\title{
Apical and basal auxin sources pattern shoot branching in a moss
}

4 Mattias Thelander ${ }^{1}$, Katarina Landberg ${ }^{1}$, Arthur Muller ${ }^{2,3}$, Gladys Cloarec ${ }^{2,4}$, Nik Cunniffe ${ }^{5}$,

5 Stéphanie Huguet ${ }^{6,7}$, Ludivine Soubigou-Taconnat ${ }^{6,7}$, Véronique Brunaud ${ }^{6,7}$ and Yoan

6 Coudert ${ }^{2, *}$

7

$8{ }^{1}$ Department of Plant Biology, Swedish University of Agricultural Sciences, The Linnean Centre for 9 Plant Biology in Uppsala, SE-750 07, Uppsala, Sweden

10 'Laboratoire Reproduction et Développement des Plantes, Université de Lyon, ENS de Lyon, UCB 11 Lyon 1, CNRS, INRA, INRIA, Lyon 69007, France

$12{ }^{3}$ Experimental Biology Research Group, Institute of Biology, Faculty of Sciences, University of 13 Neuchâtel, 2000 Neuchâtel, Switzerland

$14{ }^{4}$ Institut Jean-Pierre Bourgin, INRAE, AgroParisTech, Université Paris-Saclay, 78000 Versailles, France $15{ }^{5}$ Department of Plant Sciences, University of Cambridge, Downing Street, Cambridge CB2 3EA, UK

16 Université Paris-Saclay, CNRS, INRAE, Univ Evry, Institute of Plant Sciences Paris-Saclay (IPS2), 1791405 Orsay, France.

$18{ }^{7}$ Université de Paris, CNRS, INRAE, Institute of Plant Sciences Paris-Saclay (IPS2), 91405, Orsay, 19 France 


\section{Abstract}

23 Shoot branching mechanisms where branches arise in association with leaves - referred to 24 as lateral or axillary branching - evolved by convergence in the sporophyte of vascular plants and the gametophyte of bryophytes, and accompanied independent events of plant architectural diversification ${ }^{1}$. Previously, we showed that three hormonal cues, including auxin, have been recruited independently to co-ordinate branch patterning in flowering plant leafy shoots and moss gametophores (Coudert, Palubicki et al., 2015) ${ }^{2-4}$. Moreover, auxinmediated apical dominance, which relies on local auxin production, has been proposed as a unifying molecular regulatory mechanism of branch development across land plants ${ }^{5}$. Whilst our previous work in the moss Physcomitrium patens has gathered indirect evidence supporting the notion that auxin synthesized in gametophore apices regulates branch formation at a distance ${ }^{2}$, direct genetic evidence for a role of auxin biosynthesis in gametophore branching control is still lacking. Here, we show that gametophore apex decapitation promotes branch emergence through massive and rapid transcriptional reprogramming of auxin-responsive genes and altering auxin biosynthesis gene activity. Specifically, we identify a subset of $P$. patens TRYPTOPHAN AMINO-TRANSFERASE (TAR) and YUCCA FLAVIN MONOOXYGENASE-LIKE (YUC) auxin biosynthesis genes expressed in apical and basal regions of the gametophore, and show that they are essential for branch initiation and outgrowth control. Our results demonstrate that local auxin biosynthesis coordinates branch patterning in moss and thus constitutes a shared and ancient feature of shoot architecture control in land plants. 
43

44

45

46

47

48

49

50

51

52

53

54

55

56

57

58

59

60

61

62

63

64

65

66

67

68

69

70

71

72

73

74

75

76

77

\section{Introduction}

Understanding the mechanisms that determine the shape of living organisms is a central goal of biology. In plants, branching patterns are a major determinant of shape diversity ${ }^{6,7}$. Indeed, most plant shoots have the capacity to develop indeterminately, which allows for the continuous production of new growth axes, or branches, throughout their lifetime ${ }^{7}$. Apical dominance, the inhibitory effect exerted by shoot apices on the initiation or outgrowth of distant lateral buds, is a fundamental regulatory mechanism of branching form development. It was first described in vascular plants and is mediated, at least partly, by the production of the phytohormone auxin at the dominant shoot apex ${ }^{3,8}$. Studies in mosses have shown that excision of the gametophore (i.e. the gametophytic leafy shoot) tip - referred to as decapitation - triggers de novo branch formation ${ }^{2,9}$, indicating that apical dominance is conserved beyond vascular plants. Using the moss Physcomitrium patens as a model, we have shown that decapitation-induced branching can be alleviated by the application of auxin-impregnated lanolin on the stump of decapitated gametophores ${ }^{2}$. We have further demonstrated the inhibitory effect of auxin on branch formation by quantifying the branching patterns of $P$. patens short internodes 2-1 (shi2-1) mutants that lack a putative transcriptional regulator of YUCCA FLAVIN MONOOXYGENASE-LIKE (YUC) auxin biosynthesis genes ${ }^{10,11}$, SHI1 over-expressors and wild-type gametophores treated with natural and synthetic auxins ${ }^{2}$. We have also used a computational modelling approach to investigate whether a regulatory mechanism of branch initiation by auxin-mediated apical dominance could account for observed branch distribution patterns in wild-type and mutant gametophores ${ }^{2}$. Whilst our model supports the notion that local auxin production in the main gametophore apex and branch apices patterns branch formation at the whole-shoot level, direct genetic evidence for local auxin production in branch apices is lacking and a role for auxin biosynthesis genes in the regulation of gametophore branching has not been demonstrated yet. More generally, the relative contribution of auxin to gametophore branching is poorly understood and nonhormonal regulatory mechanisms are yet to be identified. In this study, we took advantage of the possibility to trigger branch formation by gametophore decapitation in the moss $P$. patens to address these fundamental biological questions.

\section{$\underline{\text { Results }}$}

Massive and rapid reprogramming of early auxin-responsive genes follows gametophore decapitation

To first assess the dynamics of branch emergence following decapitation, we isolated gametophores from 4-week-old wild-type colonies, cut off their apices and followed the 
development of branches over 48 hours. The first emerged branches were clearly visible 24 hours after decapitation (h.a.d.) and branches with well-developed leaves could be observed 48 h.a.d., suggesting that the earliest molecular events leading to branching occur within a day (Figure 1A). To capture a comprehensive overview of the transcriptional changes associated with early branch development, we next analyzed the transcriptome of whole gametophores collected 2, 6, 12 and 24 h.a.d., and used gametophores collected immediately after decapitation (0 h.a.d.) as a reference. The proportion of genes whose transcription was affected increased up to 12 h.a.d., leading to a maximum of 5028 and 4734 genes down- and up-regulated, respectively, suggesting that major transcriptional changes occur before the first branches become visible (Table S1, Figure S1 and S2). Gene ontology (GO) term analyses identified distinct enriched functional categories in both datasets (Table S2), but auxin-related GO terms were not significantly enriched, which was unexpected as published data suggest an important role for auxin in gametophore branching control ${ }^{2}$. To specifically address the contribution of auxin in decapitation-induced branching, we sought to directly compare the effect of auxin and decapitation at the transcriptional level. To this end, we sequenced the transcriptome of 4-week-old wild-type gametophores treated with indole-3-acetic-acid (IAA) for a short time period (30 min) and used mock-treated plants as a reference to identify early auxin-responsive genes. We found that 3652 and 4524 genes were down- and up-regulated by IAA, respectively (Table S1). By cross-referencing decapitation and IAA-regulated gene sets, we observed a striking anti-correlation between both treatments (Figure 1B). Sixty-four percent of genes down-regulated 2 h.a.d. were IAAinduced, whilst only $5 \%$ were IAA-repressed. A similar trend was observed for "6 h.a.d." (IAAinduced genes, 61\% ; IAA-repressed genes, 6\%) and "12 h.a.d." (IAA-induced genes, 51\% ; IAA-repressed genes, 8\%) gene sets. For example, both ARF and AUX/IAA auxin signalling genes, previously identified as early auxin-responsive genes ${ }^{12}$, responded oppositely in that all the genes but one were induced by IAA and repressed after decapitation (Figure 1C). Inversely, $46 \%$ of genes up-regulated 2 h.a.d. were IAA-repressed, whilst only $14 \%$ were IAA-induced, with a similar trend for "6 h.a.d." (IAA-repressed genes, 49\% ; IAA-induced genes, 8\%) and "12 h.a.d." (IAA- repressed genes, 34\% ; IAA-induced genes, 11\%) gene sets (Figure 1B). Thus, the opposite effect of decapitation and auxin application on the overall gametophore transcriptional response support the notion that the main gametophore apex is a major auxin source. Taken together, our data indicate that early auxin-responsive genes play a major role in the decapitation response.

\section{Decapitation rapidly alters TAR and YUC auxin biosynthesis gene expression}


113 We reasoned that our dataset would provide a reference to identify genes controlling apical 114 dominance, both in an auxin-dependent and independent manner (see Discussion). In this

115 context, we sought to test further the hypothesis that gametophore apices are sites of auxin

116 biosynthesis. Auxin is derived from tryptophan (TRP)-independent and dependent 117 biosynthetic pathways ${ }^{13}$. In the latter, the dominant pathway in plants, TRP is converted to 118 indole-3-pyruvic acid (IPyA) by TRYPTOPHAN AMINO-TRANSFERASE (TAA/TAR) enzymes, 119 that is then converted to auxin by YUCCA FLAVIN MONOOXYGENASE-LIKE (YUC) 120 enzymes ${ }^{14-17}$. Auxin metabolite profiling in PpTAR and PpYUC overexpression lines, and 121 pptar mutants have suggested that both steps of auxin biosynthesis are essential and 122 identified TRP-to-IPyA conversion as a rate-limiting step in $P$. patens ${ }^{13,18,19}$. To determine the 123 changes in auxin biosynthesis gene activity during decapitation-induced branching, we 124 therefore retrieved PpTAR (named TAR hereafter for the sake of readability) and PpYUC 125 (named YUC hereafter) expression profiles from our RNA-seq data. We identified two TAR 126 and three YUC genes significantly differentially expressed after decapitation (Figure 1D and 127 1E). Specifically, TARA (Pp3c21_15370V3.1), TARC (Pp3c17_6500V3.1) and YUCF 128 (Pp3c3_20490V3.1) were induced as early as 2 h.a.d., YUCC (Pp3c1_11500V3.1) was 129 induced from 12 h.a.d., and YUCB (Pp3c11_11790V3.1) was repressed. We also found that 130 the expression of TARA, YUCE (Pp3c13_21970V3.1) and YUCF, but not TARC, YUCB and 131 YUCC, was affected by exogenous auxin, suggesting that auxin may to some extent regulate 132 its own biosynthesis.

TAR and YUC genes are active in the tip, branch apices and basal portion of gametophores

136 To determine the expression domain of TARA, TARC, YUCB, YUCC and YUCF genes, we 137 analysed the activity of transcriptional fusions between corresponding promoter regions and 138 a GFP-GUS chimeric protein transformed in a wild-type background (Figure S3). Two to three independent transgenic lines were observed for each genetic construct (Table S4). We found 140 that the spatial expression domains of TAR and YUC promoters largely overlapped in whole gametophores. Beta-glucuronidase (GUS) activity was detected at the main apex, at the base, notably in rhizoid cells, and in discrete regions of the stem corresponding to initiating branch apices (Figure 2A, 2D, 2G, 2J and 2M). At the main apex, TAR promoter activity was observed in the apical cell and emerging leaves, as reported previously ${ }^{18}$, and both TAR and YUC promoters were highly active in axillary hair cells surrounding the gametophore apical cell, reminiscent of the expression pattern of other auxin-related genes like PpSHI1 and PpSHI2 ${ }^{10}$

147 (Figure $2 \mathrm{P}$ and $2 \mathrm{Q}$ ). Promoter activity of $Y U C F$, and to a lesser extent $Y U C B$ and $Y U C C$, was 
also detected in axillary hair cells elsewhere on the stem (Figure $2 \mathrm{M}, 2 \mathrm{R}$ ). This indicates that the expression of TARA and TARC is more restricted in space than that of YUCB, YUCC and $Y U C F$, and it is therefore unlikely that auxin biosynthesis occurs in leaf axils in the absence of TARA or TARC activity.

To determine how decapitation affected the activity of selected TAR and YUC transcriptional reporters, gametophores were collected 24 hours after apex excision and stained for GUS activity, thereby leaving enough time for new branches to initiate and grow out. The intensity of GUS accumulation patterns remained overall similar to intact controls, except for the YUCB promoter that seemed more weakly expressed after decapitation, likely reflecting its transcriptional repression (Figure 1E). The spatial distribution of these patterns was changed in leaf axils where we observed an activation of TARA, TARC, YUCB, YUCC and YUCF expression associated with the formation of new branches. The activity of TARA and TARC promoters was detected from the earliest stage of branch formation, throughout the branch initium (Figures 2B, 2C, 2E and 2F). In contrast, YUCB, YUCC and YUCF promoter activity were first detected at a later stage when the first leaves were clearly visible, and mostly in hair cells of the branch apical region (Figures $2 \mathrm{H}, 2 \mathrm{I}, 2 \mathrm{~K}$ ). At a more mature stage of branch development, i.e. when leaves and brown rhizoids were well-developed, TARA, TARC, YUCB, YUCC and YUCF genes were active in overlapping spatial domains mirroring those described previously in whole gametophores (Figures 2I, $2 \mathrm{~L}$ and $2 \mathrm{O}$, data not shown for TARA and $T A R C)$. The temporal delay between TAR and YUC gene activation and the differences in their spatial expression domains at early stages of branch initiation suggest that IPyA might be transported from the meristematic cells where it is synthesized to adjacent hair cells to be converted to auxin by YUC enzymes. Alternatively, these differences might be explained by the short-range movement of TAR or YUC mRNA, or corresponding proteins; the involvement of YUC genes co-expressed with TARA and TARC but not differentially expressed in our RNA-seq dataset, such as YUCE (Figure S4); or unmask a putative YUC-independent function of IPyA. Together, the above data suggest that TRP-dependent auxin biosynthesis occurs primarily in the main apex and the basal portion of gametophores, and resumes locally in leaf axils when new branches initiate.

\section{TAR genes prevent systematic branch initiation}

To investigate the function of TARA and TARC genes in gametophore branching, we grew tara, tarc and tarac knock-out mutants ${ }^{18}$ for 5 or 7 weeks and quantified their branching patterns, as done previously ${ }^{2}$. We found that tara single mutants had similar phenotypes to wild-type control plants. There was moderate evidence ${ }^{20}$ that tarc branch density was lower 
183

184

185

186

187

188

189

190

191

192

193

194

195

196

197

198

199

200

201

202

203

204

205

206

207

208

209

210

211

212

213

214

215

216

217

than control plants in older gametophores, indicating that tarc single mutants were also very similar to wild-type (Figures $3 \mathrm{~A}-3 \mathrm{C}, 3 \mathrm{E}-3 \mathrm{G}$ and $3 \mathrm{~K}-3 \mathrm{~N}$ ). In contrast, there was strong evidence that tarac double mutants had a shorter apical inhibition zone and inter-branch distance, and an increased branch density with respect to wild-type plants, suggesting a reduced apical dominance and lateral inhibition from branch meristems (Figure 3D, 3H, 3K, $3 \mathrm{~L}$ and 30 ). We also observed that the position of the lowermost branch was closer to the gametophore base in tarac mutants than in wild-type (Figure 3P), and the number of branches in the three most basal metamers was much higher in tarac mutants ( $n=23$ branches) than in tara $(n=2)$, tarc $(n=2)$ or wild-type $(n=5)$ gametophores, suggesting that local TAR activity prevents branching at the gametophore base (Figures 3A-3D). Despite the overall increase in branch number in tarac mutants, we observed strong disparities in the branch distribution pattern between gametophores. Whilst some gametophores bore branches on up to six consecutive metamers (see gametophore \#1 in Figure 3D), which has never been reported elsewhere before, others had few or no visible branches at all, which was rather counterintuitive (Figure 3D). However, on closer inspection of tarac gametophores, we noticed that tiny buds were nested in nearly every single leaf axil lacking a visible branch (Figure 3l-3J). This suggests that branch initiation was almost systematically de-repressed in tarac mutants while subsequent outgrowth was inhibited. Consistent with observed TARA and TARC expression patterns in whole gametophores and emerging branches, our data indicate that both genes act redundantly in the apices and basal portion of gametophores and their activity is necessary to prevent branch initiation and co-ordinate branch patterning.

\section{YUC genes suppress branching}

Then, to determine the role of decapitation-induced YUC genes in gametophore branching, we grew yucc, yucf and yuccf knock-out mutants ${ }^{18}$ for 5 or 7 weeks and quantified their branching patterns ${ }^{2}$ (Figure S5). Whilst the apical inhibition zone size was increased in yucc gametophores, the distance to the closest branch, the position of the lowermost branch of yucc and yucf gametophores were not changed with respect to wild-type controls (Figure 4A-4C, 4E-4G, 4I-4M). We observed slight perturbations of the branch distribution patterns in yucc and yucf single mutants, mostly explained by the branching patterns of older gametophores (Fig 4L-M). In contrast, we found strong evidence that yuccf double mutants had a reduced apical inhibition zone and inter-branch distance, and moderate evidence of an increased branch density with respect to wild-type plants (Figure 4D, 4H, 4I-4K, 4N). Moreover, the position of the lowermost branch was closer to the gametophore base in yuccf double mutants than in wild-type (Figure 3P), and the number of branches in the three most 
basal metamers was higher in yuccf mutants ( $n=15$ branches) than in yucc $(n=11)$, yucf $(n$

$219=6)$ or wild-type $(n=3)$ gametophores. Unlike tarac mutants, we did not observe emerging

220 buds in the leaf axils of yuccf gametophores. However, we sometimes noticed

221 supernumerary branches growing from the base of lateral branches in yucc mutants,

222 consistent with YUCC expression at the base of branches (Figure 4F) and indicating a local

223 inhibitory effect of YUCC. Moreover, branches seemed less developed in both single and

224 double yuc mutants than in wild-type plants. Together, the above data coincide with

225 observed expression patterns of YUC transcriptional reporters and indicate that YUCC and

226 YUCF act redundantly to inhibit branch formation but promote subsequent growth, hence

227 supporting the hypothesis of a local role of auxin biosynthesis in branch patterning.

\section{Discussion}

230 This work primarily aimed to determine the relative importance of auxin in gametophore branching and test the hypothesis that apically synthesized auxin patterns branch distribution. Using an RNA-seq approach, we show that early auxin-responsive genes have a major contribution to the transcriptional response of gametophores to decapitation. We also provide evidence that several TAR and YUC genes, together responsible for the enzymatic conversion of tryptophan to auxin, are active in the main apex and branch apices, and the base of gametophores ${ }^{13,18}$, and identify a prominent and dual role for TARA and TARC in branch initiation and growth. First, our data show that apical auxin sources suppress branch initiation at a distance. Indeed, observed perturbations of branching patterns in tarac and yuccf mutants (i.e. the reduction of the apical inhibition zone size and distance between closest branches, and the increase of branch density) qualitatively match the predicted outcome of our published computational model of gametophore branching control by hormonal cues where a reduction of auxin production in the main and lateral apices promotes branching $^{2}$. Interestingly, the model has also identified that a basal inhibitory cue is locally required to prevent branching, and genetic experiments previously led us to propose that this cue is a CCD8-derived molecule belonging to the strigolactone family of compounds ${ }^{2}$. Expression patterns of selected TAR and YUC transcriptional reporters indicate that auxin biosynthesis occurs in this region, and basal branching is strongly increased in tarac and yuccf double mutant gametophores, suggesting that auxin may act concomitantly with the CCD8-derived cue to fulfil this inhibitory role. Determining whether and how these two hormonal cues interact will require further investigation. Second, our data indicate that an adequate level of TAR and YUC activity at branch initiation sites is essential to drive branch 
253 mutants, than in wild-type. These observations corroborate the finding that tarac 254 gametophores have stunted growth $^{18}$ and suggest that low auxin biosynthesis in 255 gametophore apices hinders apical cell proliferative activity. It has previously been reported 256 that excessive auxin accumulation in gametophore tissues may also disrupt apical cell 257 function and suppress phyllid (i.e. the gametophytic leaf) emergence at the apex ${ }^{21}$, which 258 supports the idea that cellular auxin homeostasis must be tightly controlled to ensure proper 259 gametophore growth. Third, despite two whole-genome duplications in $P$. patens and their 260 possible consequences on gene redundancy ${ }^{22}$, it must be stressed that deleting only 2 out 261 of 4 TARs, and 2 out of 6 YUCs was sufficient to reveal the function of both gene families in 262 gametophore branching control. Nevertheless, it cannot be excluded that other TAR or YUC 263 genes may also be involved in this process.

264 From an evolutionary perspective, TAR-YUC-mediated auxin biosynthesis originated in 265 streptophyte algae and is conserved in land plants ${ }^{23,24}$. The genome of the liverwort 266 Marchantia polymorpha contains only one functional TAA gene, named MpTAA, and two YUC 267 genes, named MpYUC1 and MpYUC2 ${ }^{25}$. Similar to our observations in moss gametophores, 268 MpYUC2 expression domain is broader than that of MpTAA in the liverwort thallus (i.e. the 269 gametophytic body), and both domains overlap in meristematic notches that contain the 270 apical stem cells at the origin of thallus tissues. Although thallus branching occurs by notch 271 dichotomy, a type of apical branching that is developmentally distinct from gametophore 272 branching ${ }^{7,26}$, Marchantia taa and yuc knock-down plants also display hyperbranching 273 phenotypes. Beyond bryophytes, various studies in the flowering plant Arabidopsis thaliana 274 have shown that AtTAA1/WEI8, AtTAR2, AtYUC1, AtYUC4 and AtYUC6 genes are expressed 275 in discrete and partially overlapping domains of the shoot apical meristem ${ }^{14,15}$. Double and 276 triple mutants in these genes, including yuc1yuc4, yuc1yuc4yuc6 and taa1tar2 allele 277 combinations, show various degrees of decreased apical dominance reflected by an 278 enhanced production of branches. Together, this suggests that lateral and apical branching 279 modes evolving separately in flowering plant sporophytes, and moss and liverwort 280 gametophytes, are underpinned by a shared and ancient molecular mechanism driving local 281 auxin biosynthesis in shoot meristematic structures, which represents a novel discovery of 282 deep homology in land plants ${ }^{21,27,28}$.

283 The functional analysis of PpTAR and PpYUC auxin biosynthesis genes demonstrated that 284 our RNA-seq approach has the potential to identify novel regulators of gametophore 285 branching. Moreover, the relatively small differences in gene expression detected at the 286 whole gametophore level (see for example Figure 1D-E) likely under-estimate the magnitude 287 of regulatory changes occurring locally, since genes underpinning branch initiation are 
expressed in restricted spatial domains. In the future, our dataset will help us to decipher

289 how auxin is integrated at the molecular level to regulate branching. For instance, GO

290 analyses revealed a significant enrichment of the "DNA binding transcription factor activity"

291 term in gene sets up- and down-regulated after decapitation (Table S2). Members of the

292 APETALA2/ETHYLENE RESPONSIVE FACTOR (AP2/ERF) family represented about two

293 thirds of identified DNA binding transcription factor coding genes and included putative

294 conserved regulators of meristematic function, such as STEMIN1 (Pp3c1_27440v3.1), known

295 to promote stem cell formation through local depletion of a repressive chromatin mark, and

296 Pp3c26_9880v3.1, the closest homologue to Solanum lycopersicum LEAFLESS and $A$.

297 thaliana PUCHI and DORNRÖSCHEN-LIKE, which are regulated by auxin and involved in

298 meristem identity and lateral organ initiation control ${ }^{29-32}$. We also found that GO terms related

299 to "trehalose metabolism" were significantly enriched in the "up-regulated 2 h.a.d." gene set.

300 Interestingly, trehalose 6-phosphate (T6P) metabolic genes regulate branching and apical

301 dominance in flowering plants ${ }^{33-35}$. For example, shoot decapitation in Pisum sativum (pea)

302 triggers an increase of T6P levels in a few hours, which contributes to promoting branch

303 outgrowth ${ }^{33}$. T6P is synthesized from uridine diphosphate-glucose and glucose-6-phosphate

304 by the activity of T6P SYNTHASES (TPS) and converted to trehalose by T6P

305 PHOSPHATASES (TPP), which is then hydrolysed into glucose by TREHALASES (TRE).

306 Transgenic Arabidopsis thaliana plants with increased TPP activity in axillary buds have

307 reduced T6P levels and delayed branch outgrowth, whilst plants with increased levels of T6P

308 in the vasculature have enhanced branching ${ }^{34}$. Moreover, mutations in Zea mays RAMOSA3

309 (RA3) and its paralog ZmTPP4 that both encode TPP enzymes, lead to increased

310 inflorescence branching ${ }^{35,36}$. In Physcomitrium, we found that all PpTPS genes were

311 repressed after decapitation, whilst PpTPP and PpTRE genes were either repressed or

312 induced (Figure S6), suggesting that T6P levels are dynamically regulated during

313 decapitation-induced branching. Besides, we found that TPS genes were IAA-induced and

314 decapitation-repressed, and GO terms related to trehalose metabolism were associated with

315 genes both induced by auxin and up-regulated after decapitation (Table S3), consistent with

316 TPP and TRE expression profiles 2 and 6 h.a.d. (Figure S6). This suggests that apically-

317 produced auxin regulates the activity of T6P metabolic genes in the gametophore, which has

318 not been evidenced yet in flowering plants. Further studies will be needed to determine the

319 biological function of T6P metabolic genes and AP2/ERF DNA-binding transcription factors,

320 and explore their crosstalk with auxin in the moss $P$. patens. Finally, it must be stressed that

321 our dataset contains a large number of differentially expressed genes with uncharacterized 
322 function, representing an untapped reservoir of novel putative regulators of shoot 323 architecture.

\section{Material and methods}

\section{Physcomitrium patens plant growth and transformation}

328 The Reute ${ }^{37}$ strain of Physcomitrella patens was used as the wild-type (WT) background for generating all the transgenic lines, the Gransden strain being largely sterile in laboratory conditions. Moss colonies were initiated from $1 \mathrm{~mm}^{2}$ spot cultures and cultivated in sterile Magenta GA-7 tissue culture vessels (Bioworld, Dublin, OH, USA) on BCDAT medium (250 $\mathrm{mg} / \mathrm{L} \mathrm{MgSO}{ }_{4} .7 \mathrm{H}_{2} \mathrm{O}, 250 \mathrm{mg} / \mathrm{L} \mathrm{KH}_{2} \mathrm{PO}_{4}$ (pH 6.5), $1010 \mathrm{mg} / \mathrm{L} \mathrm{KNO}, 12.5 \mathrm{mg} / \mathrm{L}, \mathrm{FeSO}_{4} .7 \mathrm{H}_{2} \mathrm{O}$, $0.001 \%$ Trace Element Solution $\left(0.614 \mathrm{mg} / \mathrm{L} \mathrm{H}_{3} \mathrm{BO}_{3}, 0.055 \mathrm{mg} / \mathrm{L} \mathrm{AlK}\left(\mathrm{SO}_{4}\right)_{2} .12 \mathrm{H}_{2} \mathrm{O}, 0.055\right.$ $\mathrm{mg} / \mathrm{L} \mathrm{CuSO}{ }_{4} .5 \mathrm{H}_{2} \mathrm{O}, 0.028 \mathrm{mg} / \mathrm{L} \mathrm{KBr}, 0.028 \mathrm{mg} / \mathrm{L} \mathrm{LiCl}, 0.389 \mathrm{mg} / \mathrm{L} \mathrm{MnCl} 2.4 \mathrm{H}_{2} \mathrm{O}, 0.055 \mathrm{mg} / \mathrm{L}$ $\mathrm{CoCl}_{2} .6 \mathrm{H}_{2} \mathrm{O}, 0.055 \mathrm{mg} / \mathrm{LnSO}_{4} .7 \mathrm{H}_{2} \mathrm{O}, 0.028 \mathrm{mg} / \mathrm{L} \mathrm{KI}$ and $\left.0.028 \mathrm{mg} / \mathrm{L} \mathrm{SnCl} 2.2 \mathrm{H}_{2} \mathrm{O}\right), 0.92 \mathrm{~g} / \mathrm{L}$ di-ammonium tartrate $\left(\mathrm{C}_{4} \mathrm{H}_{12} \mathrm{~N}_{2} \mathrm{O}_{6}\right)$ and $8 \mathrm{~g} / \mathrm{L}$ agar with $\mathrm{CaCl}_{2}$ added to a $1 \mathrm{mM}$ concentration after autoclaving, at $23^{\circ} \mathrm{C}$ under a $16 \mathrm{~h}$ light/8h dark cycle, at $50-150 \mu \mathrm{mol} . \mathrm{m}^{-2} \cdot \mathrm{s}^{-1}$ in MLR-352 growth cabinets (PHCbi, Etten-Leur, The Netherlands).

\section{Decapitation and auxin treatment}

341 For pharmacological treatments, a $70 \%$ ethanol solution containing $100 \mathrm{mM}$ indole-3-acetic acid (IAA) (Merck KGaA, Darmstadt, Germany) was diluted 10,000 times in water to reach a final concentration of $10 \mu \mathrm{M}$ IAA. A $70 \%$ ethanol solution was diluted 10,000 times in water and used as a mock control. Moss cultures were soaked in diluted solutions for 30 minutes before tissue harvest. For decapitation, gametophores were teased apart from moss colonies with thin tip tweezers, planted in an upright position on BCDAT petri dishes and their apical portion was cut off with micro-scissors, as described in Coudert, Palubicki et al. (2015)².

\section{Generation of YUC transcriptional reporters and yuc mutants}

350 All the transgenic lines used in this study are listed in Table S4. Generation and confirmation of the transcriptional reporter lines TARA::GFP-GUS, TARC::GFP-GUS, and YUCF::GFPGUS, as well as the knockout lines tara, tarc, tarac, yucc, and yucf have been previously described $^{18}$. To produce the YUCB::GFPGUS reporter construct pMT244 (Figure S3), a 2090 bp YUCB promoter fragment was amplified from gDNA with primers SS235/SS236 and trimmed with $\mathrm{BamHI} / \mathrm{Ncol}$. The resulting fragment was cloned between the BamHI/Ncol sites 
357 subsequent integration into the neutral Pp108 locus ${ }^{38}$. Similarly, to produce the

358 YUCC::GFPGUS reporter construct pMT251 (Figure S3), a 1767 bp YUCC promoter fragment

359 was amplified from gDNA with primers SS237/SS238, trimmed with BamHI/BspHI, and

360 cloned between the same two sites in pMT211. Both reporter constructs were linearized with

361 Sfil before they were transformed into WT protoplast as previously described ${ }^{39}$. Stable

362 transformants were selected in the presence of $50 \mu \mathrm{g} \cdot \mathrm{ml}^{-1}$ hygromycin (Duchefa H0192;

363 Haarlem, the Netherlands). Correct integration was confirmed by PCR (Figure S3). For primer

364 sequences, see Table S5. The yuccf double knockout lines were produced by a sexual cross

365 of the confirmed single mutants Ppyucc-2 and Ppyucf-1 described previously ${ }^{18}$, according to

366 the method presented in Thelander et al. ${ }^{38}$. Double knockout lines were confirmed by PCRs

367 demonstrating the loss of internal gene sequences (Figure S5).

369 GUS staining and plant imaging

370 Physcomitrium patens gametophores were isolated from colonies grown on BCDAT and 371 incubated at $37^{\circ} \mathrm{C}$ in a $100 \mathrm{mM}$ phosphate buffer $(\mathrm{pH} 7)$ with $10 \mathrm{mM}$ Tris $(\mathrm{pH} 8), 1 \mathrm{mM}$ EDTA 372 (pH 8), $0.05 \%$ Triton $\mathrm{X}-100,5 \mathrm{mM}$ potassium ferricyanide, $5 \mathrm{mM}$ potassium ferrocyanide and $3732 \mathrm{mM}$ X-GlcA (5-Bromo-4-chloro-3-indolyl- $\beta$-D-glucuronic acid), using times indicated in

374 Figure 2. Tissues were de-stained in $70 \%$ ethanol and imaged with a Keyence VHX-900F 375 digital microscope with a 5-50 X or a 50-200 X objective. Plants not subject to GUS staining 376 were imaged in the same conditions.

377

\section{Definition of branches in Physcomitrium patens}

379 According to Coudert et al. ${ }^{1}$, a module is defined as "a portion of gametophore arising from 380 a single apical cell". Here, we consider that the main gametophore axis corresponds to a Class I module, and gametophore branches correspond to Class II lateral modules.

\section{RNA-seq data production and analysis}

384 For each biological replicate and condition, RNA were pooled from 5-10 gametophores 385 (Gransden ${ }^{40}$ strain). Three independent biological replicates were produced. Total RNA was extracted using an RNeasy Plant Mini Kit (Qiagen, Hilden, Germany) and treated with DNAse according to the supplier's instructions. RNA-seq libraries were made using the TruSeq_Stranded_mRNA_SamplePrep_Guide_15031047_D protocol (Illumina, California, 
392

393

394

395

396

397

398

399

400

401

402

403

404

405

406

407

408

409

410

411

412

413

414

415

416

417

418

419

420

421

422

423

424

425

bar-coded adapters, generating approximately 30 millions of PE reads per sample. All experimental steps, from growth conditions to bioinformatic analyses, were deposited in the CATdb database ${ }^{41}$ (http://tools.ips2.u-psud.fr/CATdb/, ProjectID NGS2017_09_Moss1) according to the MINSEQE 'minimum information about a high-throughput sequencing experiment' (https://doi.org/10.25504/FAIRsharing.a55z32). To facilitate comparisons, all samples were processed similarly from trimming to count. RNA-Seq pre-processing included trimming library adapters and performing quality controls. Raw data (fastq) were trimmed with fastx toolkit for Phred Quality Score Qscore $>20$, read length $>30$ bases, and ribosome sequences were removed with sortMeRNA ${ }^{42}$. The mapper Bowtie ${ }^{43}$ (version 2) was used to align reads against the Physcomitrium (Physcomitrella) patens transcriptome (with local option and other default parameters). The 32926 genes were extracted from Phytozome database (Physcomitrella patens transcripts v3.1 gene model). The abundance of each gene was calculated by a local script which parses SAM files and counts only paired-end reads for which both reads map unambiguously one gene, removing multi-hits. According to these rules, around $82,7 \%$ of PE reads were associated to a gene, 5-6\% PE reads were unmapped and $12-13 \%$ of PE reads with multi-hits were removed. Choices for the differential analysis were made based on the article by Rigaill et $a^{44}$. Genes which did not have at least one read after a counts per million (CPM) normalization in at least one half of the samples were discarded. Library size was normalized using the TMM method and count distribution was modeled with a negative binomial generalized linear model. Dispersion was estimated by the edgeR method ${ }^{45}$ (version 1.12.0) in the statistical software ' $R$ ' (http://www.R-project.org, version 2.15.0). Expression differences were compared between " 0 h.a.d." and "2 h.a.d.", "6 h.a.d.", "12 h.a.d." or "24 h.a.d." conditions for the "decapitation experiment", and between samples "mock" and "10 $\mu \mathrm{M}$ IAA" conditions for the "auxin experiment", using likelihood ratio test and $p$-values were adjusted by the Benjamini-Hochberg procedure to control FDR. A gene was considered as differentially expressed if its adjusted p-value was lower than 0.05 . Venn diagrams were generated using InteractiVenn ${ }^{46}$. Gene Ontology (GO) term enrichment analyses were performed with ShinyGO (version 0.66$)^{47}$.

\section{RNA-seq data availability}

RNA-seq datasets are available in the international repository GEO (Gene Expression Omnibus ${ }^{48}$, http://www.ncbi.nlm.nih.gov/geo) under the project identifier GSE188843 (token = ctslsswyfjchdcp).

\section{Sample-size estimation and replicates}


426 For data shown in Figure 1, three independent biological replicates were produced for each

427 condition, and each replicate corresponded to RNA extracted from 5 to 10 gametophores.

428 For Figure 2, two or three independent transgenic lines were analysed for TAR and YUC

429 transcriptional reporters. Independent lines transformed with the same genetic construct

430 showed similar GUS staining patterns. Numbers in panels $A, D, G, J$ and $M$ indicate the

431 proportion of gametophores with a GUS staining pattern similar to the picture. For Figure 3,

432 data shown in panels A-D were obtained from 30 gametophores collected 5 weeks after

433 protonema inoculation and 30 gametophores collected 7 weeks after protonema inoculation,

434 metrics in panels $\mathrm{K}, \mathrm{L}$ and $\mathrm{P}$ could be calculated only for gametophores with more than one

435 branch, data in panels $\mathrm{M}-\mathrm{O}$ correspond to all gametophores. For Figure 4, data shown in

436 panels A-D were obtained from 30 gametophores collected 5 weeks after protonema 437 inoculation and 30 gametophores collected 7 weeks after protonema inoculation, data in

438 panels I-K could be calculated only for gametophores with more than one branch, data in 439 panels L-N correspond to all gametophores.

440

\section{Statistical analyses}

442 Generalised linear modelling was used to test whether the relationship between branch 443 number and gametophore length depended on genotype (File S1). Poisson or negative 444 binomial regression was used, depending on whether the data were over-dispersed, i.e. 445 whether the data were more variable than would be expected under a Poisson model. The 446 most complex fitted models were of the form $\log (\mathbb{E}(B \mid L, X))=a_{0}+a_{1} X+\left(a_{2}+a_{3} X\right) L$, in 447 which $a_{0}, a_{1}, a_{2}$ and $a_{3}$ are coefficients, $L$ is the length, $X$ is an indicator variable depending 448 on genotype (i.e. $X$ took values of either 0 or 1 depending upon whether the mutant or WT 449 was considered), and $\mathbb{E}(B \mid L, X)$ is the expected branch number $(B)$ at any given pair of 450 values of $L$ and $X$. Analysis of deviance and backwards stepwise elimination was used to find 451 the minimal model that received support from the experimental data. A mutant was 452 considered different from WT if either the interaction term $\left(a_{3}\right)$ or the term corresponding to 453 genotype $\left(a_{1}\right)$ remained in the minimal model, i.e. if the expected branch number was affected 454 by the value of $X$. The choice of whether to use a Poisson and negative binomial model was 455 made via a formal test of over-dispersion for the Poisson version of the model with an 456 interaction, using the function overdispersion() in the package $A \mathrm{ER}^{49}$. The terms "weak", 457 "moderate", "strong" and "very strong evidence" reflected the translation of $p$-values into the 458 language of evidence proposed by Muff et al. ${ }^{20}$ 

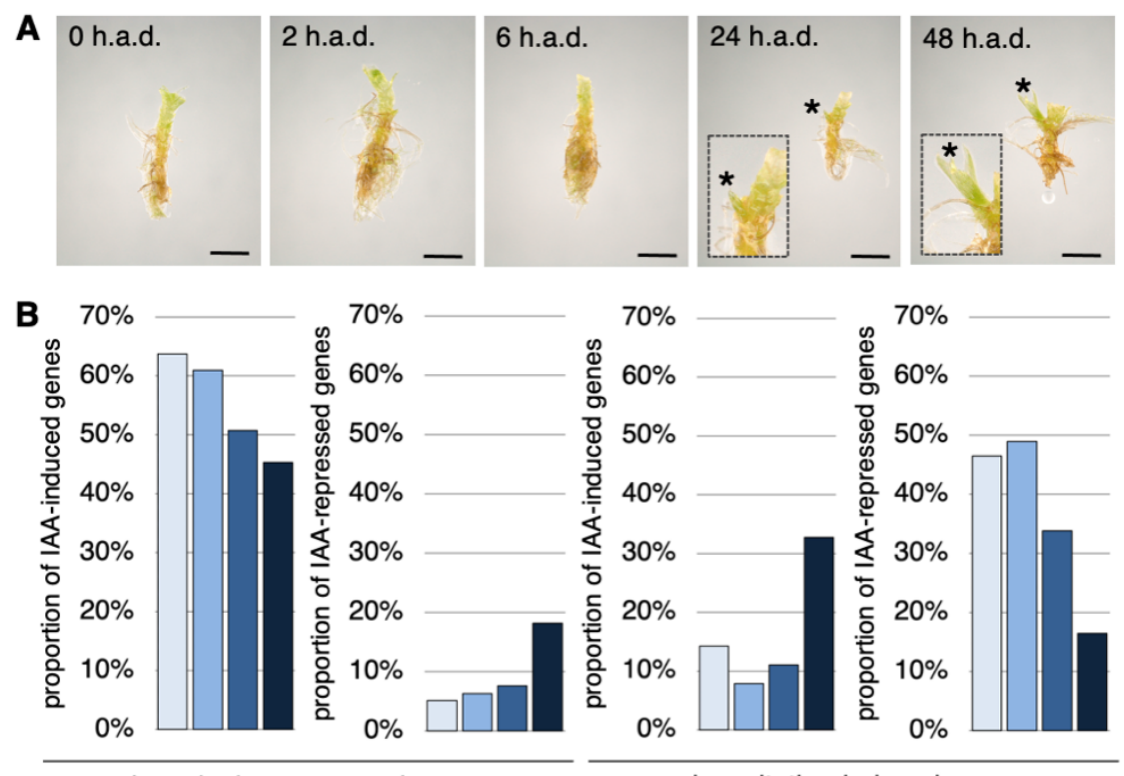

decapitation-repressed genes

decapitation-induced genes
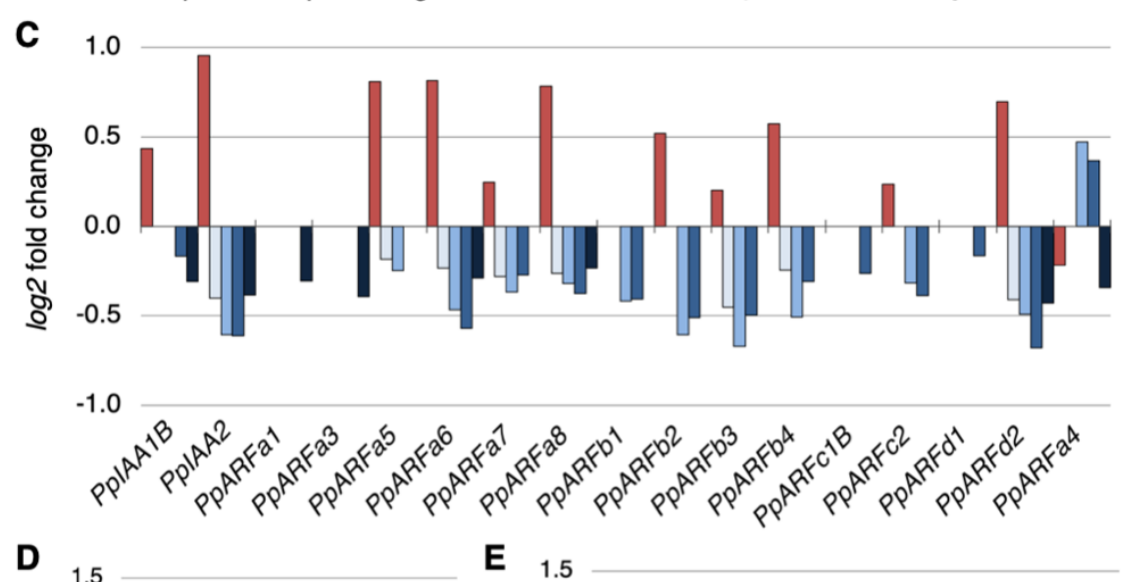

463
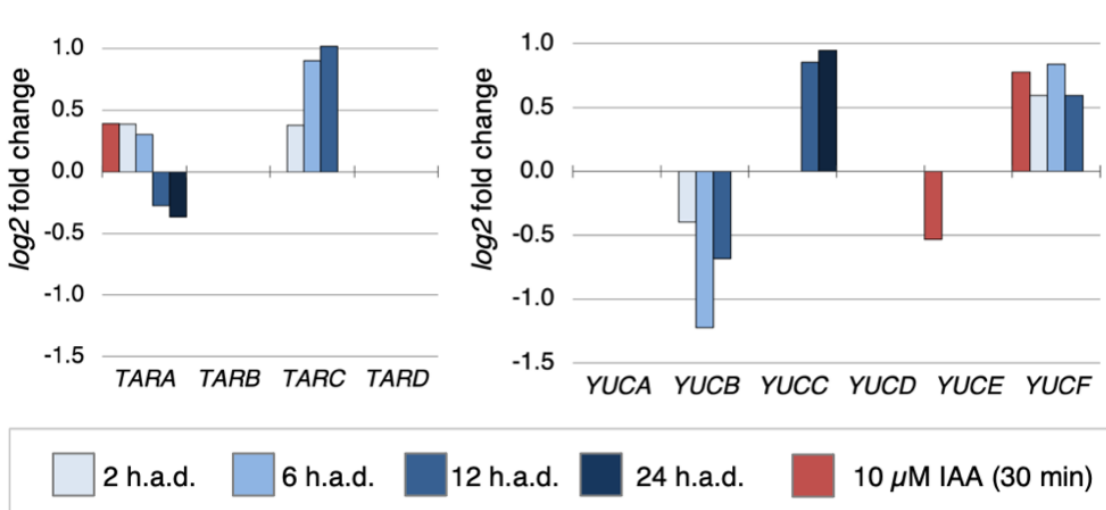

464 Figure 1. Decapitation affects the expression of early auxin-responsive genes, and

465 auxin signalling and biosynthesis genes. (A) New branches (indicated with asterisks) emerged within 24 hours after gametophore decapitation. Leaves were removed before imaging to observe gametophore branches. Insets show magnifications of emerging 
468 branches. Scale bar $=1 \mathrm{~mm}$. (B) Cross-analysis of transcriptomes of decapitated and auxin469 treated gametophores showed that about two thirds of the genes repressed 2 h.a.d. were 470 induced by a 30-minute treatment with $10 \mu \mathrm{M}$ indole-3-acetic acid (auxin), and conversely 471 nearly half of the genes induced 2 h.a.d. were repressed by auxin. (C) Bar plots showed that 472 all but one $A R F$ and $A U X / I A A$ auxin signalling genes identified were repressed after 473 decapitation and induced by auxin. (D-E) Expression of two TAR and three YUC auxin 474 biosynthesis genes was affected by decapitation. The colour code shown at the bottom of 475 the figure is used in panels B-E.

476 


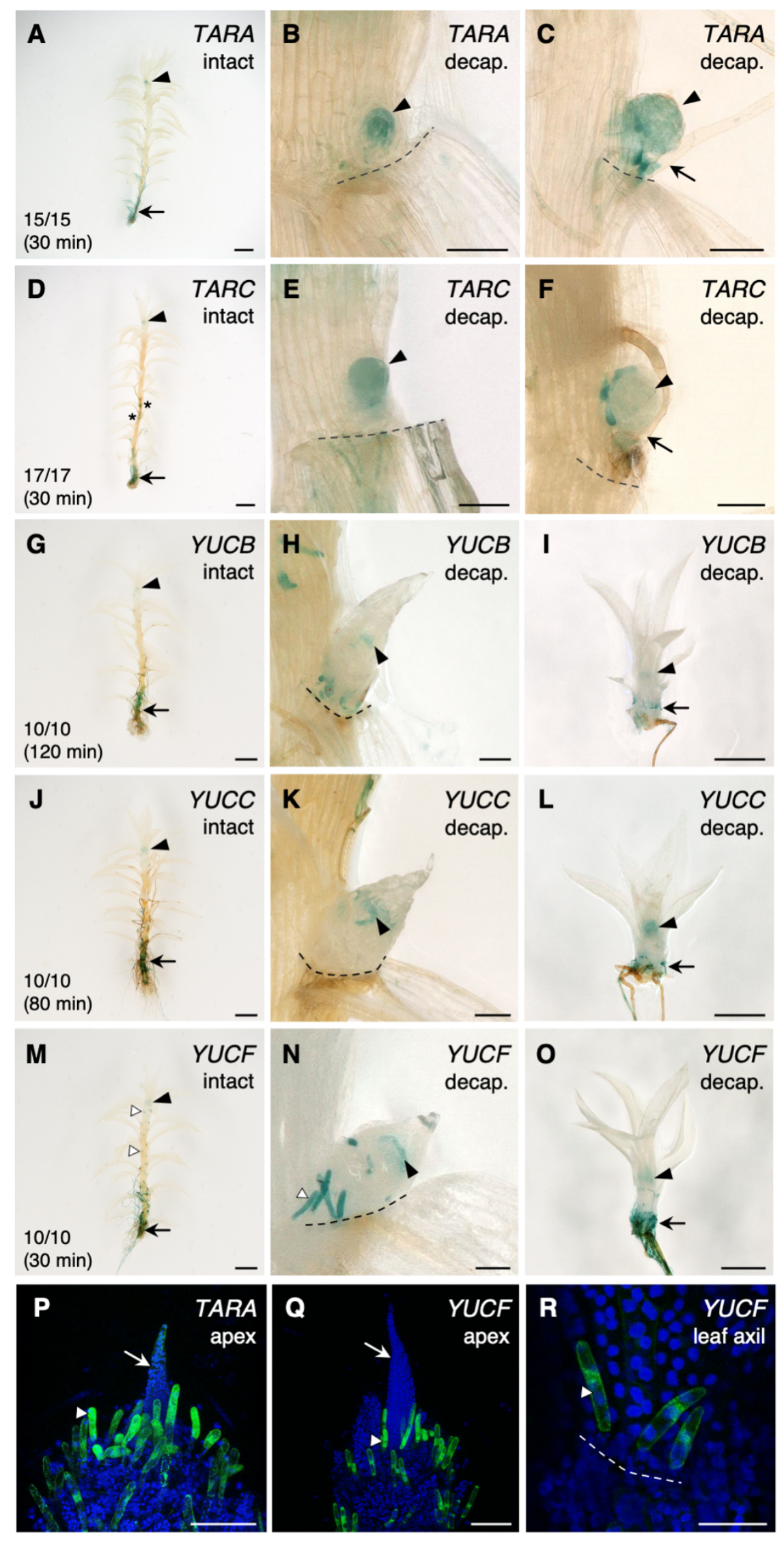

478 Figure 2. TAR and YUC genes are expressed in meristematic and basal regions of 479 Physcomitrium gametophores. (A-O) GUS staining of TARA::GFP-GUS (A-C), TARC::GFP- 
480 GUS (D-F), YUCB::GFP-GUS (G-I), YUCC::GFP-GUS (J-L) and YUCF::GFP-GUS (M-O)

481 transgenic lines revealed that TAR and YUC genes are active in largely overlapping

482 expression domains. Gametophore were either observed before (“intact”) or after ("decap.")

483 apex excision. In intact gametophores, TARA and TARC are expressed in the main apex

484 (black arrowheads), in emerging branches (asterisks) and in the basal region (black arrows)

485 (A, D). Following decapitation, TARA and TARC expression is detected in initiating lateral

486 branches (black arrowheads) but not in rhizoids (black arrows) (B, C, E, F). In intact

487 gametophores, YUCB, YUCC and YUCF are expressed in the main apex (black arrowheads)

488 and in the basal region (black arrows). YUCF expression is also observed in axillary hairs in

489 leaf axils (white arrowheads) (G, J, M). Following decapitation, YUCB, YUCC and YUCF

490 expression is first detected at a later stage than TARA and TARC. GUS staining was found

491 in axillary hairs at the apex of newly formed branches (black arrowheads) and, at a later stage,

492 in branch rhizoids (black arrows) (H, I, K, L, N, O). (P-R) GFP signal in TARA::GFP-GUS and

493 YUCF::GFP-GUS lines was strongest in hairs (white arrowheads) located the gametophore

494 apex (P-Q), and/or in leaf axils (R). Emerging leaves are indicated with white arrows. Dashed

495 lines mark the boundary between the stem and the detached leaf $(B, C, E, F, H, K, N, R)$.

496 Gametophores were soaked in GUS staining solution for times specified in panels A, D, G, J

497 and $\mathrm{M}$. The scale bars represent $1 \mathrm{~mm}$ in $\mathrm{A}, \mathrm{D}, \mathrm{G}, \mathrm{J}$ and $\mathrm{M}, 500 \mu \mathrm{m}$ in I, L, and $\mathrm{O}, 100 \mu \mathrm{m}$ in

$498 \mathrm{~B}, \mathrm{C}, \mathrm{E}, \mathrm{F}, \mathrm{H}, \mathrm{K}, \mathrm{P}$ and $\mathrm{Q}$, and $50 \mu \mathrm{m}$ in $\mathrm{N}$ and $\mathrm{R}$. 


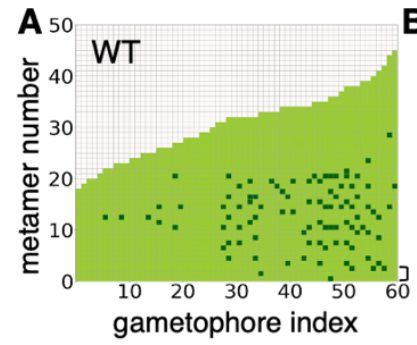

$\mathbf{E}$

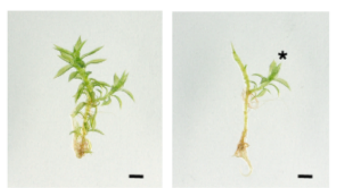

I tarac

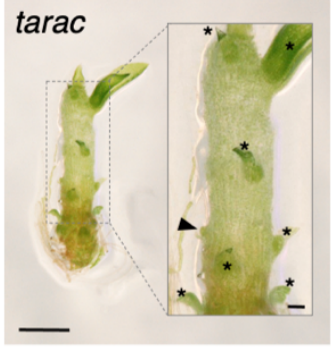

M

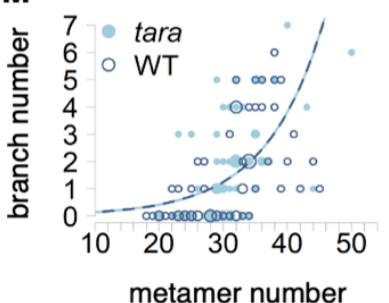

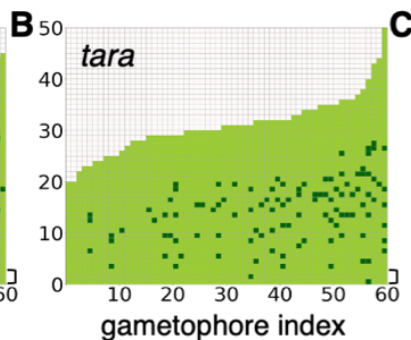

$\mathbf{F}$
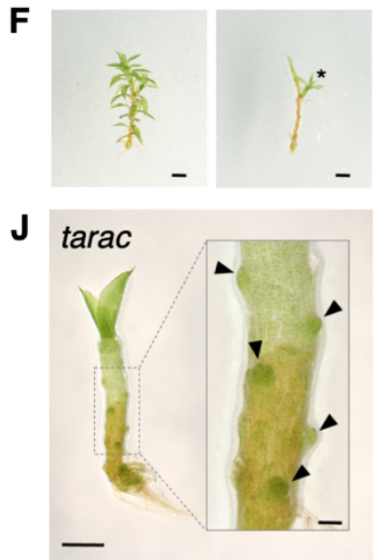

$\mathbf{N}$

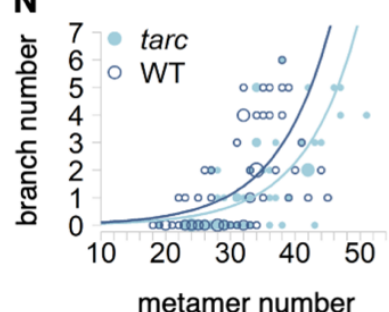

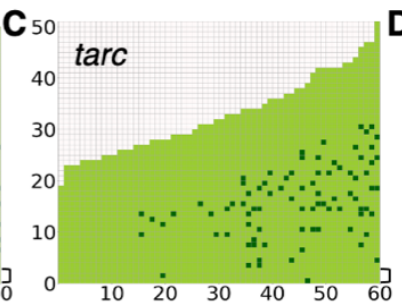

gametophore index

G
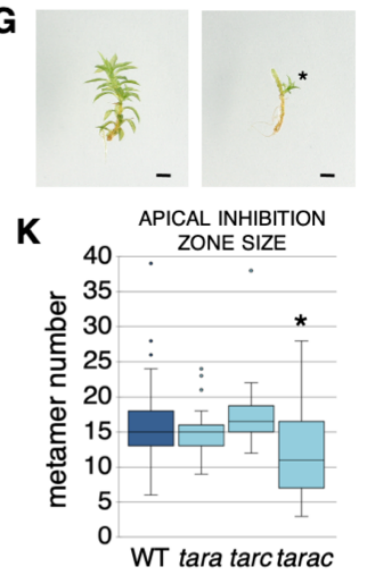

0

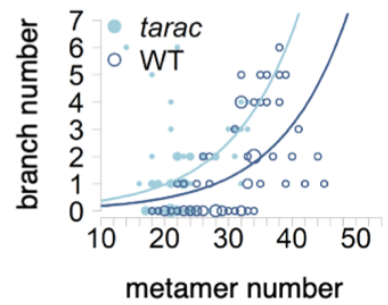

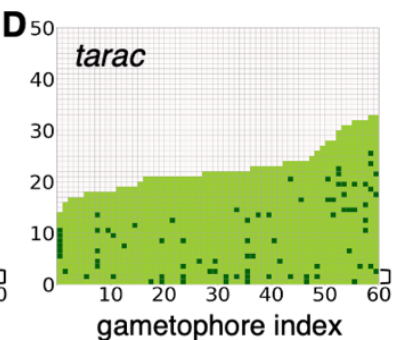

H
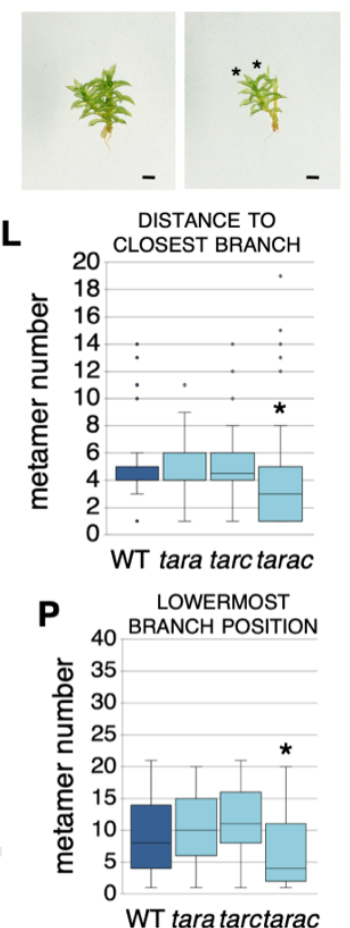

Figure 3. TARA and TARC genes prevent branch initiation and pattern branch

502 distribution along the gametophore axis. (A-D) Branching patterns in WT, tara, tarc and

503 tarac mutants are presented as in Coudert, Palubicki et al. (2015)². Brackets indicate the three

504 lowermost metamers. $(\mathrm{E}-\mathrm{H}) \mathrm{WT}(\mathrm{E})$, $\operatorname{tara}(\mathrm{F}), \operatorname{tarc}(\mathrm{G})$ and $\operatorname{tarac}(\mathrm{H})$ gametophores before (left)

505 and after (right) removing leaves, with asterisks marking branches. Scale bars represent 1

$506 \mathrm{~mm}$. (I-J) tarac gametophores with leaves removed revealed lateral buds with no visible

507 leaves (arrowheads) and tiny branches with few visible leaves (asterisks), which is never

508 observed in WT in which branches initiate de novo and grow out with no dormancy period.

509 Scale bars represent $500 \mu \mathrm{m}$ in the main panels and $100 \mu \mathrm{m}$ in the insets. (K) There was

510 strong evidence ${ }^{20}$ that the apical inhibition zone was reduced in tarac double mutants, but

511 not in tara or tarc single mutants, compared with wild-type control (Wilcoxon rank sum test

512 with continuity correction different from $\mathrm{WT}$, ${ }^{*} \mathrm{p}$-value $\leq 0.05$; WT versus tara, $\mathrm{p}$-value $=0.35$;

513 WT versus tarc, $p$-value $=0.07$; WT versus tarac, $p$-value $=0.005)$. (L) There was very strong

514 evidence that the distance to the closest branch, a measurement of branch spacing, was

515 significantly reduced in tarac double mutants, but not in tara or tarc single mutants, compared with wild-type control (Wilcoxon rank sum test with continuity correction different from WT, 
$517{ }^{*} \mathrm{p}$-value $\leq 0.05 ;$ WT versus tara, $\mathrm{p}$-value $=0.92$; WT versus tarc, $\mathrm{p}$-value $=0.14$; WT versus

518 tarac, $\mathrm{p}$-value $=0.0009) .(\mathbf{M}-\mathbf{O})$ Bubble plots showed that branch number at a given length is

519 increased in tarac, slightly decreased in older tarc gametophores, and unchanged in tara,

520 compared with WT. Gametophore length is represented as the number of metamers and the

521 bubble area is proportional to the number of gametophores with the same branch number

$522(B)$ at a given length $(L)$. The data were over-dispersed, and so negative binomial regression

523 was used to test whether and how the relationship between branch number and

524 gametophore length differed between mutants and WT (see 'Material and methods'). For (M)

525 the best-fitting relationship indicated no difference between tara and WT ( $p$-value $=0.39$;

$526 \log (\mathbb{E}(B \mid L))=-3.11+0.11 L$ for both treatments). For $(\mathrm{N})$ the best-fitting relationship

527 indicated WT was larger than tarc at all lengths $(\mathrm{p}$-value $=0.01 ; \log (\mathbb{E}(B \mid L, W))=-4.02+$

$5280.50 W+0.12 L$, where $W=1$ for WT). For $(O)$ the best-fitting relationship indicated WT was

529 smaller than tarac at all lengths ( $\mathrm{p}$-value=0.006; $\log (\mathbb{E}(B \mid L, W))=-1.94-0.73 W+0.095 L$,

530 where $W=1$ for $\mathrm{WT})$. (P) The position of the lowermost branch was closer to the

531 gametophore base in tarac than in tara or tarc in comparison with WT (Wilcoxon rank sum

532 test with continuity correction different from WT, * $p$-value $\leq 0.05$; WT versus tara, $p$-value $=$

$5330.30 ;$ WT versus tarc, $\mathrm{p}$-value $=0.12 ; \mathrm{WT}$ versus tarac, $\mathrm{p}$-value $=0.007$ ).

534 

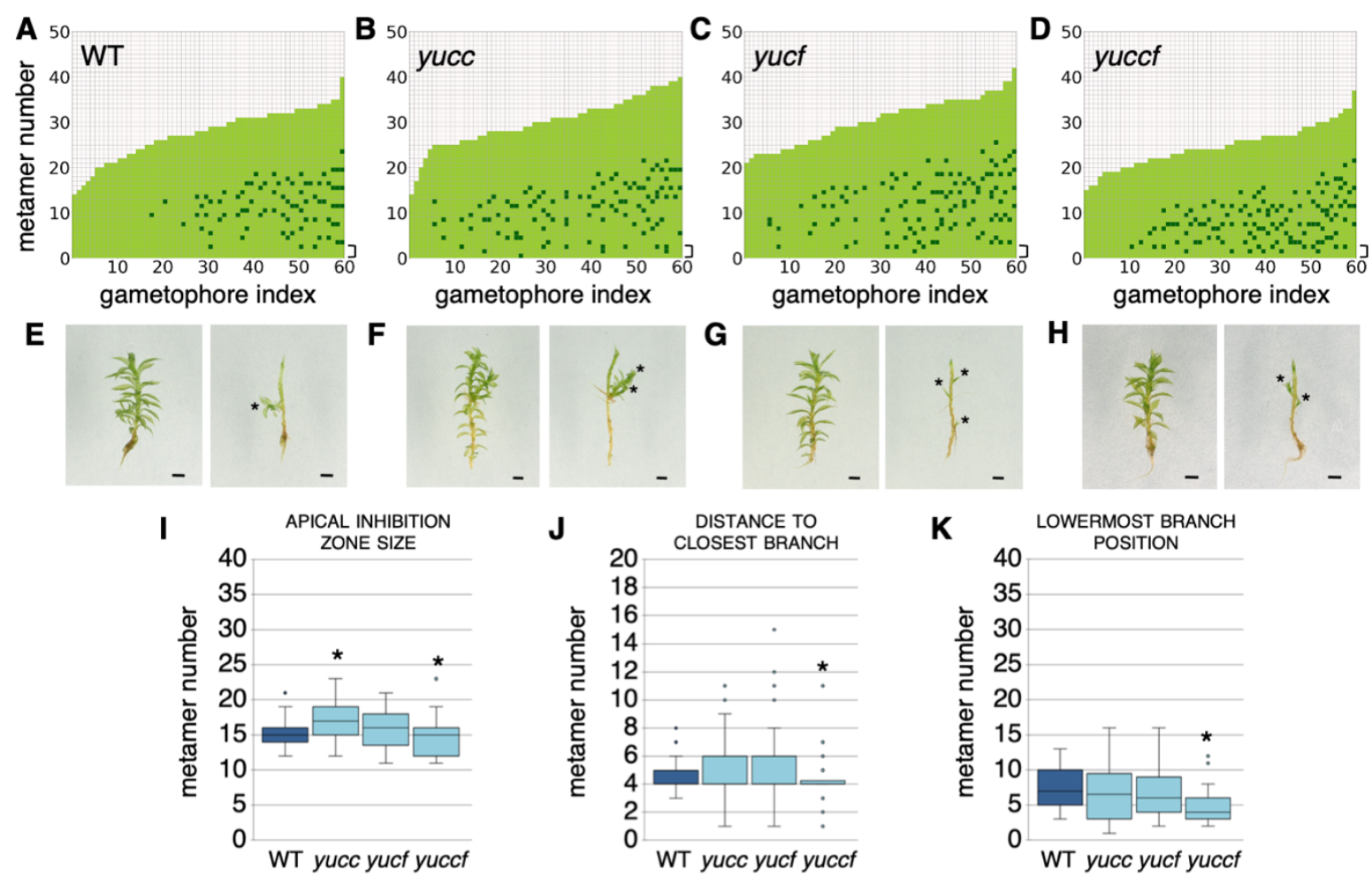

G

H
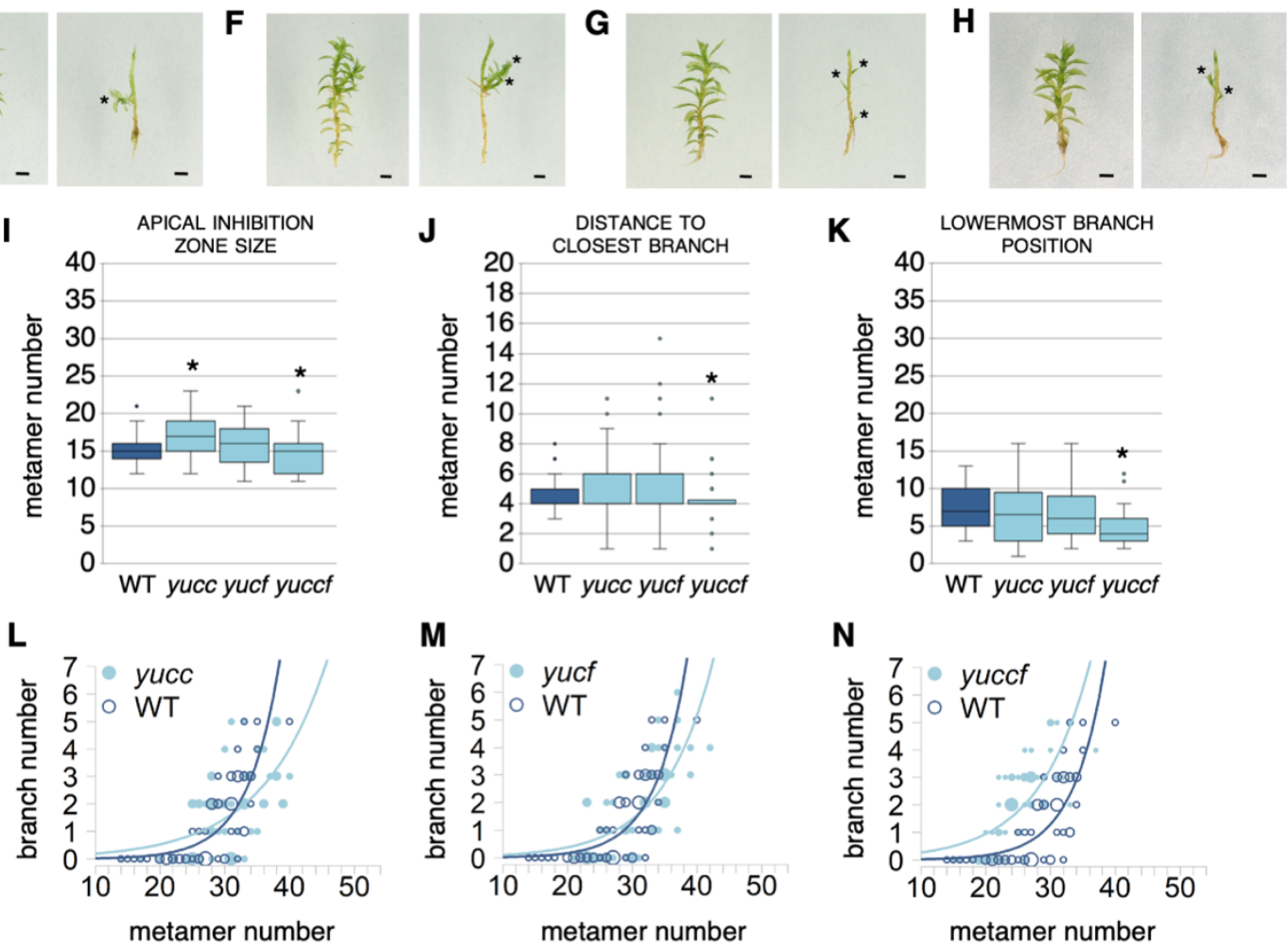

Figure 4. YUCC and YUCF genes repress branching. (A-D) Branching patterns in WT, yucC,

537 yucf and yuccf mutants. Brackets indicate the three lowermost metamers. (E-H) WT (E), yucc

$538(\mathrm{~F})$, yucf $(\mathrm{G})$ and yuccf $(\mathrm{H})$ gametophores before (left) and after (right) removing leaves, with

539 asterisks marking branches. Scale bars represent $1 \mathrm{~mm}$. (I) There was strong and moderate

540 evidence that the apical inhibition zone was different in yucc and yuccf mutants, respectively,

541 compared with wild-type control (Wilcoxon rank sum test with continuity correction different

542 from $W T$, ${ }^{*} p$-value $\leq 0.05 ; W T$ versus yucc, $p$-value $=0.003 ; W T$ versus yucf, $p$-value $=0.82$;

543 WT versus tarac, $p$-value $=0.05)$. $(\mathbf{J})$ There was strong evidence that the distance to the

544 closest branch, a measurement of branch spacing, was reduced in yuccf double mutants,

545 but not in yucc or yucf single mutants, compared with wild-type control (Wilcoxon rank sum

546 test with continuity correction different from WT, ${ }^{*} \mathrm{p}$-value $\leq 0.05$; WT versus yuc, $\mathrm{p}$-value $=$

5470.77 ; WT versus yucf, $\mathrm{p}$-value $=0.76$; WT versus yuccf, $\mathrm{p}$-value $=0.01)$. $(\mathbf{K})$ There was very

548 strong evidence that the position of the lowermost branch was closer to the gametophore

549 base in yuccf than in yucc or yucf in comparison with WT (Wilcoxon rank sum test with

550 continuity correction different from $W T$, ${ }^{*} p$-value $\leq 0.05$; WT versus yucc, $p$-value $=0.49$; 
551 WT versus yucf, $\mathrm{p}$-value $=0.34$; WT versus yuccf, $\mathrm{p}$-value $=0.0006)$. (L-M) Bubble plots

552 showed that branch number responded differently to length in all three mutants compared

553 with WT. Gametophore length is represented as the number of metamers and the bubble

554 area is proportional to the number of gametophores with the same branch number $(B)$ at a

555 given length $(L)$. The data were not over-dispersed, and so Poisson regression was used to

556 test whether and how the relationship between branch number and gametophore length

557 depended on treatment (see 'Material and methods'). The best-fitting relationship included

558 an interaction for all three mutants, indicating the nature of the difference in the number of

559 branches between WT and mutant depended upon the length (in (L), $p=0.002$,

$560 \log (\mathbb{E}(B \mid L, Y))=-5.28+3.17 Y+(0.20-0.10 Y) L$, where $Y=1$ for yucc; in (M) $\mathrm{p}=0.03$,

$561 \log (\mathbb{E}(B \mid L, Y))=-5.28+2.25 Y+(0.20-0.07 Y) L$, where $Y=1$ for yucf; in $(\mathrm{N}) \mathrm{p}=0.02$,

$562 \log (\mathbb{E}(B \mid L, Y))=-5.28+3.25 Y+(0.20-0.08 Y) L$, where $Y=1$ for yuccf). 


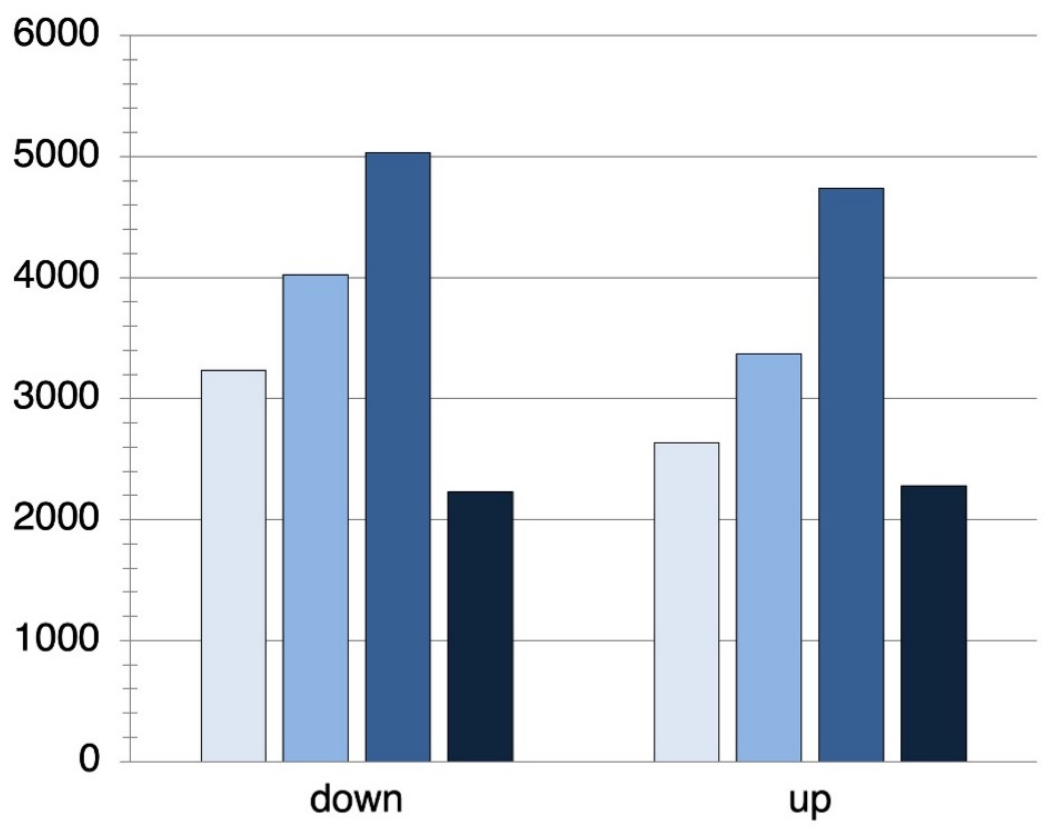

$\square 2$ h.a.d. $\square 6$ h.a.d. $\square 12$ h.a.d. $\square 24$ h.a.d.

567 Figure S1. Number of genes repressed or induced 2, 6, 12 and 24 hours after 568 decapitation. 
bioRxiv preprint doi: https://doi. org/10.1101/2022 01.04 474977· this version posted January 6, 2022. The copyright holder for this preprint (which was not certified by peer review) is the author/funder, who has granted bioRxiv a license to display the preprint in perpetuity. It is made available under aCC-BY 4.0 International license.
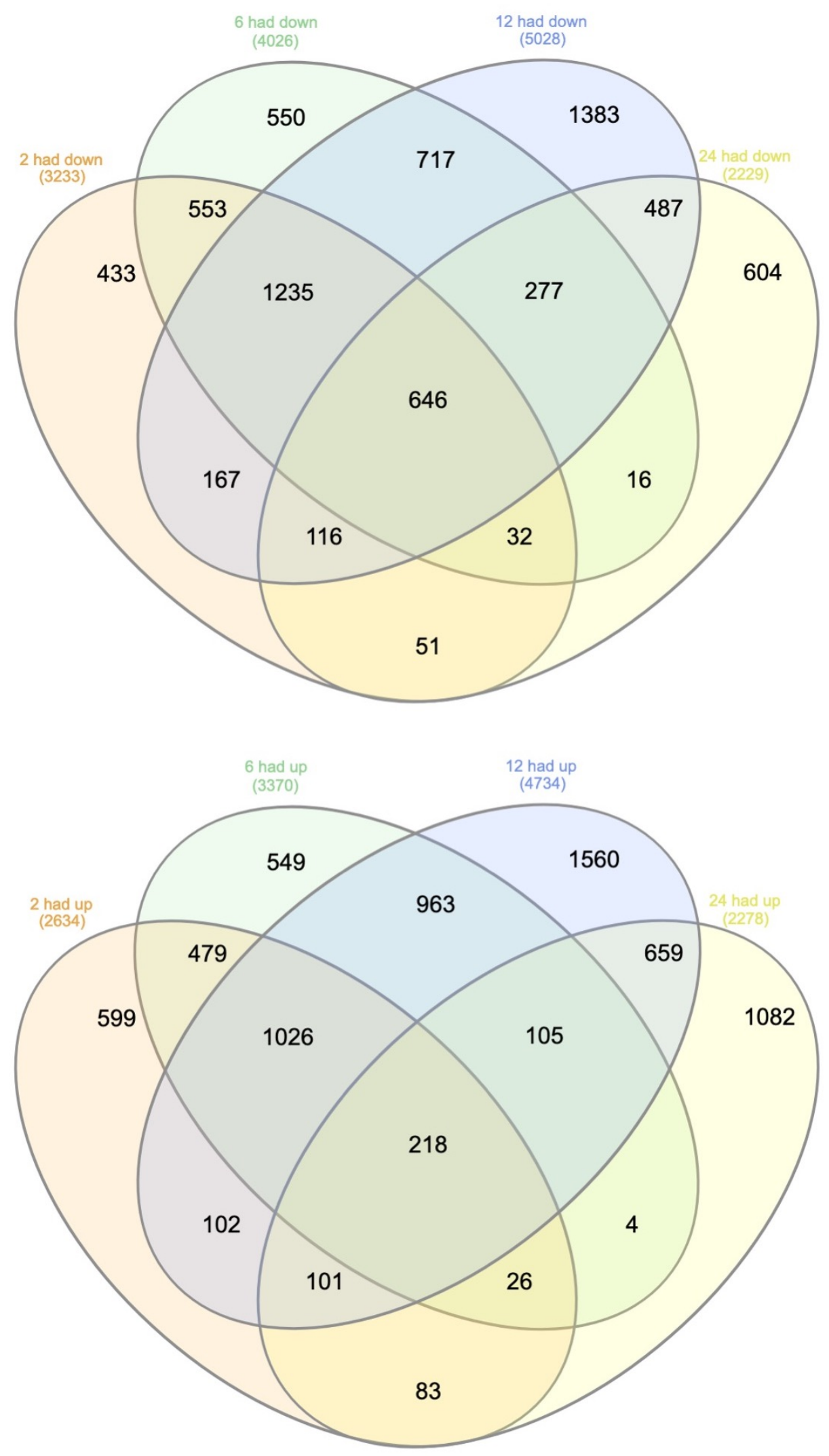

571 Figure S2. Venn diagrams for all genes repressed or induced 2, 6, 12 and 24 hours after 
A
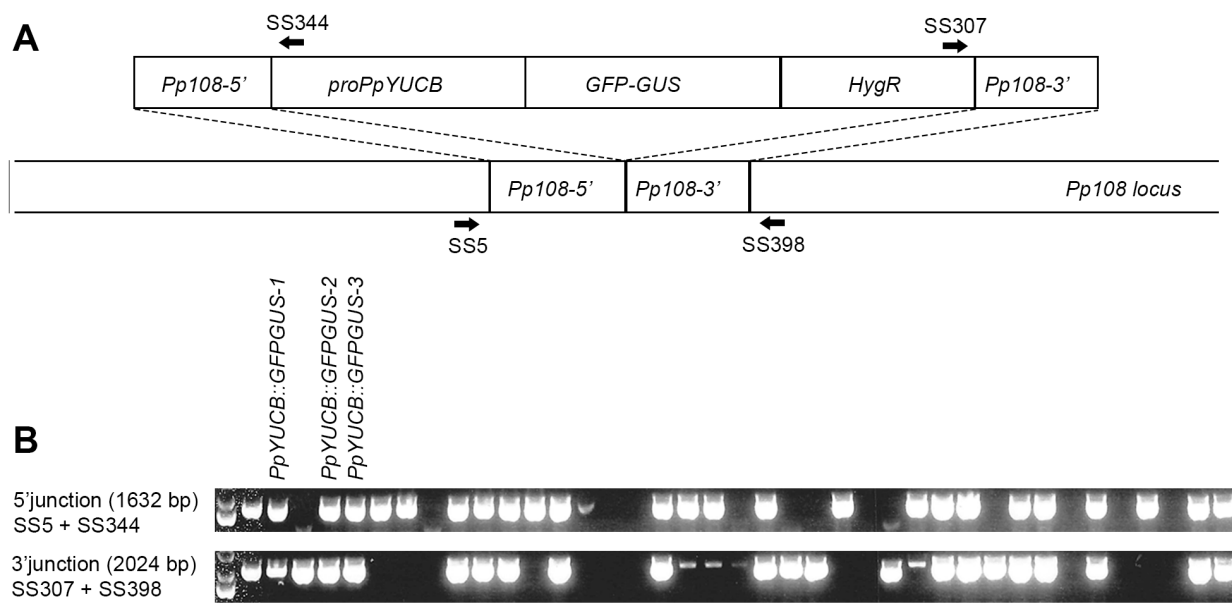

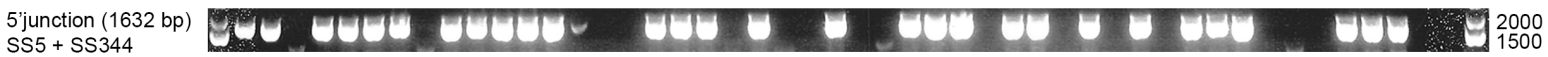
3'junction (2024 bp) S307+ SS398

C
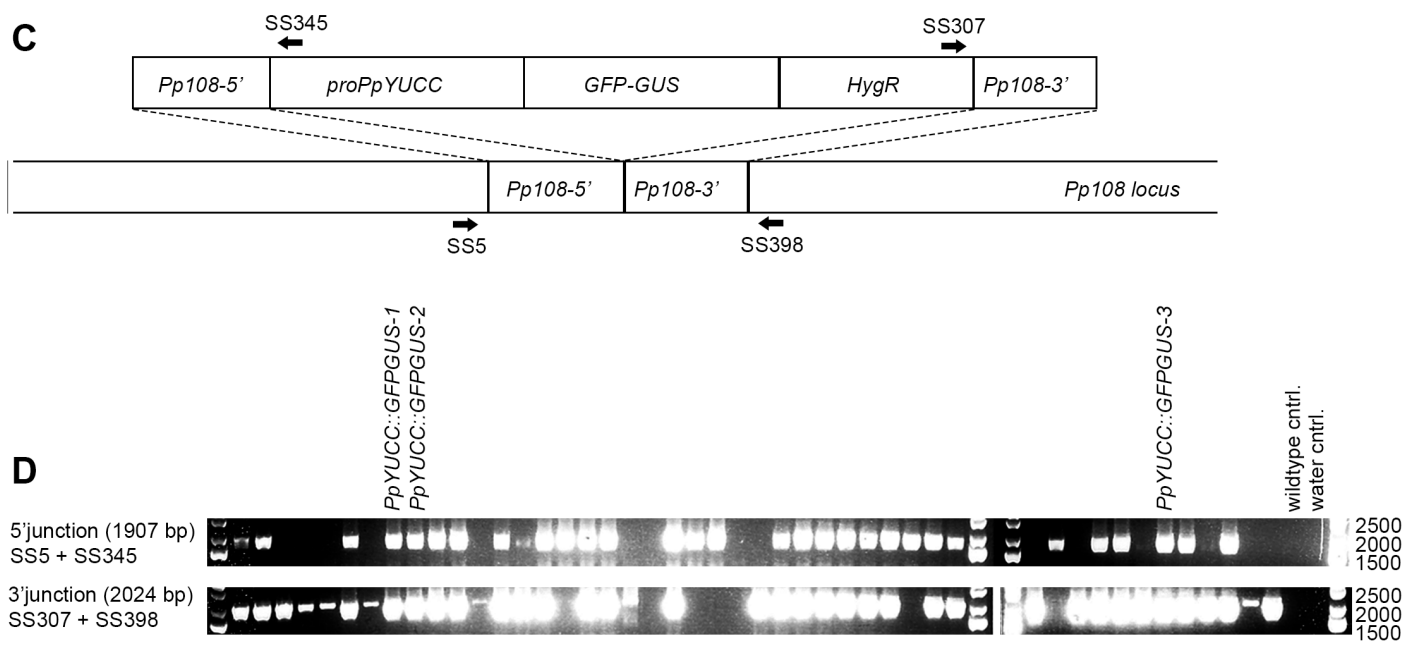

574

3'junction (2024 bp) SS307+ SS398

575

576

Figure S3. PCR verification of PpYUCB and PpYUCC transcriptional reporter lines in

577 WT background (A) Schematic view of the PpYUCB::GFPGUS reporter construct pMT244

578 and the Pp108 locus to which it was targeted. Arrows mark the approximate annealing sites

579 of primers used for PCR verification in B. (B) PCR verification of 5' and 3' junctions to confirm

580 correct integration of the PpYUCB::GFPGUS reporter construct into the Pp108 locus. (C)

581 Schematic view of the PpYUCC::GFPGUS reporter construct pMT251 and the Pp108 locus

582 to which it was targeted. Arrows mark the approximate annealing sites of primers used for

583 PCR verification in D. (D) PCR verification of 5' and 3' junctions to confirm correct integration

584 of the PpYUCC::GFPGUS reporter construct into the Pp108 locus. In both B and D, expected product sizes are indicated within parentheses. For primer sequences, see Table S5. 


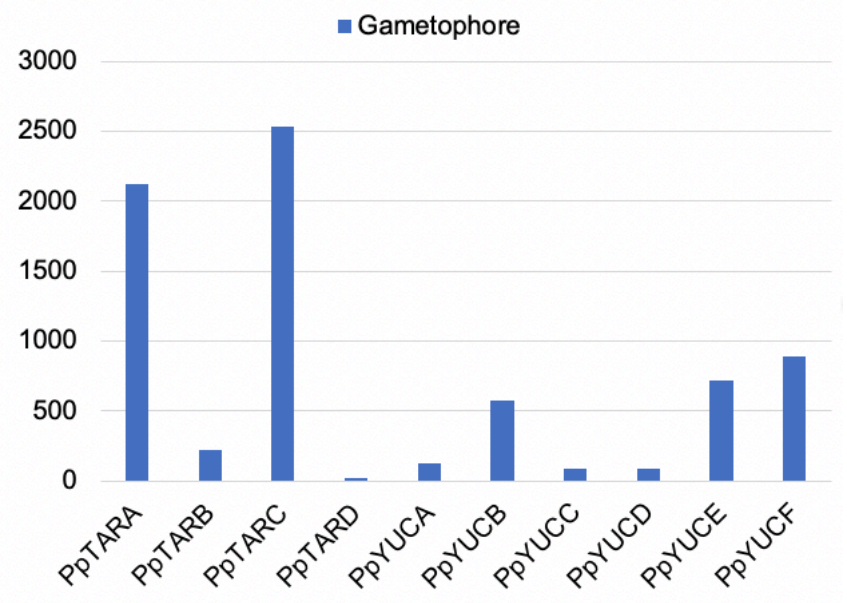

589 Figure S4. Absolute expression levels of TAR and YUC genes in Physcomitrium patens

590 gametophores retrieved from Ortiz-Ramirez et al. (2016) ${ }^{50}$. Correspondence between

591 gene names and identifiers: PpTARA (Pp3c21_15370V3.1, Pp1s167_103V6.1), PpTARB

592 (Pp3c18_15140V3.1, Pp1s3_273V6.1), PpTARC (Pp3c17_6500V3.1, Pp1s26_28V6.1),

593 PpTARD (Pp3c26_12520V3.1, Pp1s6_329V6.1), PpYUCA (Pp3c3_18590V1.1,

594 Pp1s312_60V6.1), PpYUCB (Pp3c11_11790V3.1, Pp1s11_6V6.1), PpYUCC

595 (Pp3c1_11500V3.1, Pp1s139_131V6.1), PpYUCD (Pp3c2_27740V3.1, Pp1s22_291V6.1),

596 PpYUCE (Pp3c13_21970V3.1, Pp1s37_90V6.1), PpYUCF (Pp3c3_20490V3.1,

597 Pp1s204_126V6.1).

598 


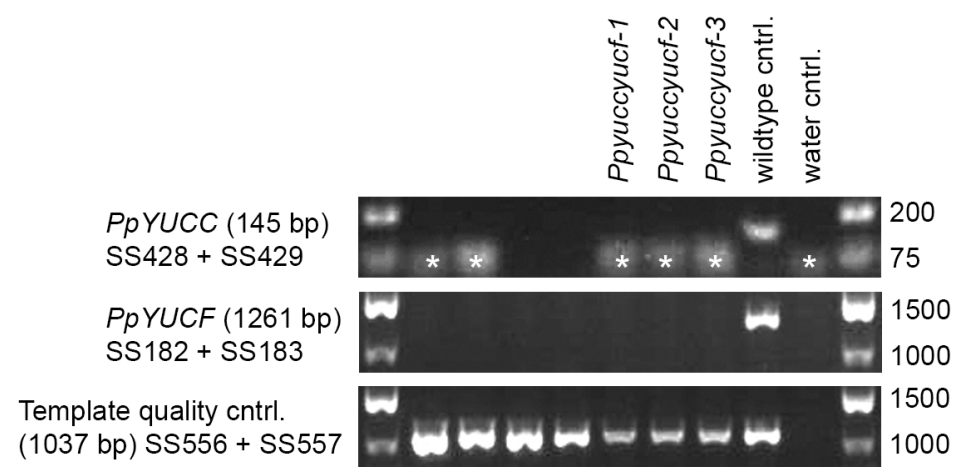

600

601 Figure S5. PCR genotyping of yuccf double knockout lines produced by a sexual cross 602 of the single knockout lines Ppyucc-2 and Ppyucf-1 (Landberg et al., 2020) ${ }^{18}$. The upper 603 panel shows the result for a PCR confirming the loss of an internal PpYUCC gene sequence. 604 Asterisks mark primer dimers. The middle panel shows the result for a PCR confirming the 605 loss of an internal PpYUCF gene sequence. The bottom panel shows amplification of an 606 unrelated locus to confirm integrity of the gDNA used as template in all three panels. Next to 607 each panel, the primers used and the expected product size are noted. For primer sequences, 608 see Table S5. 

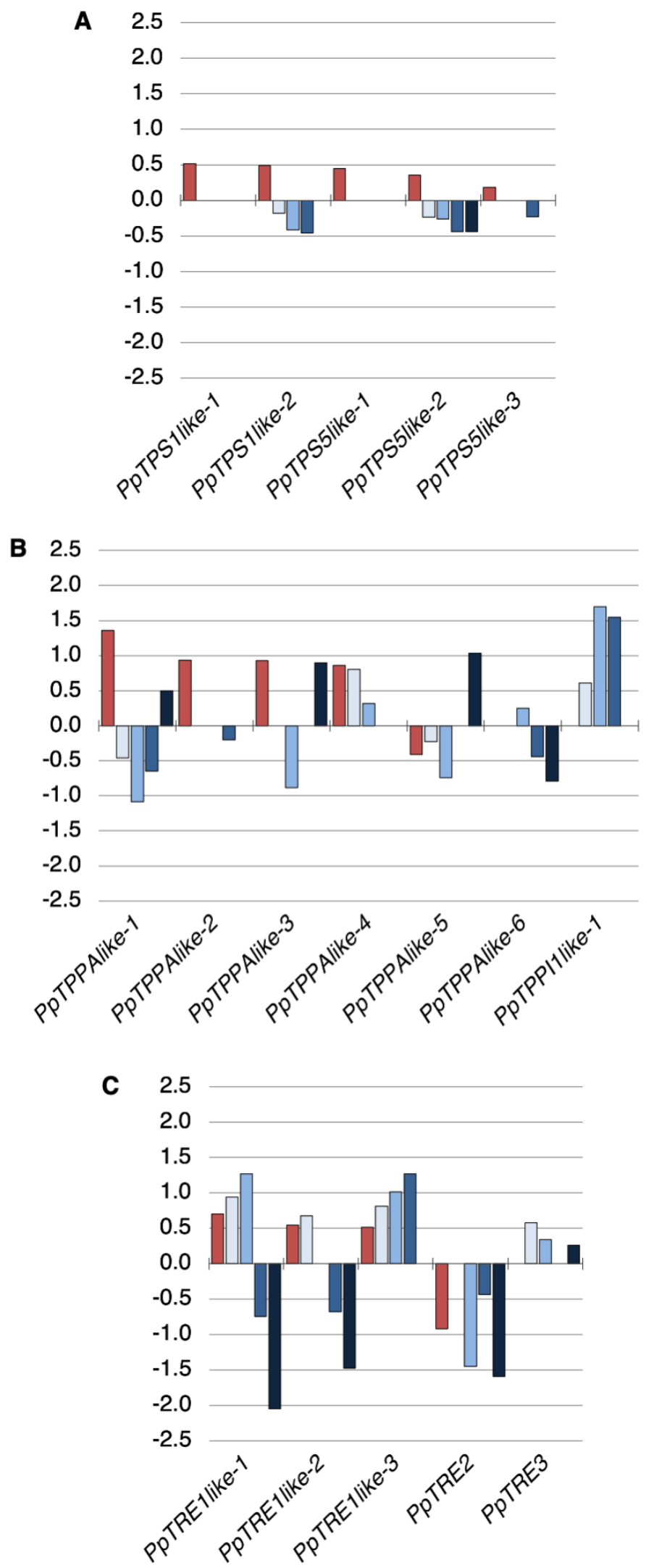

2 h.a.d. $\quad 6$ h.a.d. $\quad 12$ h.a.d.

24 h.a.d.

$10 \mu \mathrm{M}$ IAA (30 min) 
611 Figure S6. Expression of trehalose-6-phosphate (T6P) metabolism genes is affected by

612 exogenous auxin and gametophore decapitation. Bar plots show log2 fold-change in 613 expression of T6P synthase (PpTPS), T6P phosphatase (PpTPP) and trehalase (PpTRE)

614 coding genes in wild-type Physcomitrium patens gametophores after treatment with auxin 615 (red bars) or decapitation (blue bars). Correspondence between gene names and identifiers: 616 PpTPPAlike-1 (Pp3c12_20050V3.1), PpTPPAlike-2 (Pp3c4_20080V3.1), PpTPPAlike-3 617 (Pp3c4_11080V3.1), PpTPPAlike-4 (Pp3c10_13170V3.1), PpTPPAlike-5 (Pp3c3_25080V3.1), 618 PpTPPAlike-6 (Pp3c3_22990V3.1), PpTPPC1like-1 (Pp3c12_7510V3.1), PpTPPE1like-1 619 (Pp3c12_7530V3.1), PpTPPI1like-1 (Pp3c10_23130V3.1), PpTPS1like-1 (Pp3c5_17730V3.1), 620 PpTPS1like-2 (Pp3c6_16450V3.1), PpTPS5like-1 (Pp3c25_6990V3.1), PpTPS5like-2 621 (Pp3c11_17560V3.1), PpTPS5like-3 (Pp3c7_15250V3.1), PpTRE1like-1 (Pp3c5_16470V3.1), 622 PpTRE1like-2 (Pp3c23_11240V3.1), PpTRE1like-3 (Pp3c16_7830V3.1), PpTRE1like-4 623 (Pp3c24_9748V3.1), PpTRE1like-5 (Pp3c24_9750V3.1), PpTRE2 (Pp3c6_4940V3.1), PpTRE3 (Pp3c10_5310V3.1).

625

626

\section{Acknowledgements}

628 YC acknowledges Fabrice Besnard, Joe Cammarata and Teva Vernoux for constructive discussions and suggestions on a draft version of this article, Stéphanie Hallet for technical support, and the CNRS (ATIP-Avenir programme) for research funding. The sequencing platform (POPS) benefited from the support of the LabEx Saclay Plant Sciences-SPS (ANR10-LABX-0040-SPS).

633

634

\section{References}

636

637

1. Coudert, Y., Bell, N.E., Edelin, C., and Harrison, C.J. (2017). Multiple innovations underpinned branching form diversification in mosses. New Phytol 22, 810.

2. Coudert, Y., Palubicki, W., Ljung, K., Novak, O., Leyser, O., and Harrison, C.J. (2015). Three ancient hormonal cues co-ordinate shoot branching in a moss. eLife 4, e06808.

640 3. Domagalska, M.A., and Leyser, O. (2011). Signal integration in the control of shoot branching. Nat Rev Mol Cell Biol 12, 211-221.

642 4. Ferguson, B.J., and Beveridge, C.A. (2009). Roles for auxin, cytokinin, and strigolactone in regulating shoot branching. Plant Physiol 149, 1929-1944.

644 5. Coudert, Y., Harris, S., and Charrier, B. (2019). Design Principles of Branching 
6. Barthélémy, D., and Caraglio, Y. (2007). Plant architecture: a dynamic, multilevel and comprehensive approach to plant form, structure and ontogeny. Ann Bot 99, 375-407.

7. Coudert, Y. (2017). The Evolution of Branching in Land Plants: Between Conservation and Diversity. In Evolutionary Developmental Biology (Springer International Publishing), pp. 1-17.

8. Cline, M. (1997). Concepts and terminology of apical dominance. Am J Bot 84, 1064.

9. Von Maltzahn, K. (1959). Interaction between Kinetin and Indoleacetic Acid in the Control of Bud Reactivation in Splachnum ampullaceum (L.) Hedw. Nature 183, 60-61.

10. Eklund, D.M., Thelander, M., Landberg, K., Ståldal, V., Nilsson, A., Johansson, M., Valsecchi, I., Pederson, E.R.A., Kowalczyk, M., Ljung, K., et al. (2010). Homologues of the Arabidopsis thaliana SHI/STY/LRP1 genes control auxin biosynthesis and affect growth and development in the moss Physcomitrella patens. Development 137, 12751284.

11. Sohlberg, J.J., Myrenås, M., Kuusk, S., Lagercrantz, U., Kowalczyk, M., Sandberg, G., and Sundberg, E. (2006). STY1 regulates auxin homeostasis and affects apical-basal patterning of the Arabidopsis gynoecium. The Plant Journal 47, 112-123.

12. Lavy, M., Prigge, M.J., Tao, S., Shain, S., Kuo, A., Kirchsteiger, K., Estelle, M., and Hardtke, C.S. (2016). Constitutive auxin response in Physcomitrella reveals complex interactions between Aux/IAA and ARF proteins. eLife Sciences 5, e13325.

13. Thelander, M., Landberg, K., and Sundberg, E. (2018). Auxin-mediated developmental control in the moss Physcomitrella patens. J Exp Bot 69, 277-290.

14. Yadav, S., Kumar, H., and Yadav, R.K. (2020). Local auxin biosynthesis promotes shoot patterning and stem cell differentiation in Arabidopsis shoot apex. bioRxiv, 819342.

15. Galvan-Ampudia, C.S., Cerutti, G., Legrand, J., Brunoud, G., Martin-Arevalillo, R., Azais, R., Bayle, V., Moussu, S., Wenzl, C., Jaillais, Y., et al. (2020). Temporal integration of auxin information for the regulation of patterning. eLife 9, e55832.

16. Cheng, Y., Dai, X., and Zhao, Y. (2006). Auxin biosynthesis by the YUCCA flavin monooxygenases controls the formation of floral organs and vascular tissues in Arabidopsis. Genes Dev. 20, 1790-1799.

17. Ljung, K. (2013). Auxin metabolism and homeostasis during plant development. Development 140, 943-950.

18. Landberg, K., Šimura, J., Ljung, K., Sundberg, E., and Thelander, M. (2021). Studies of moss reproductive development indicate that auxin biosynthesis in apical stem cells may constitute an ancestral function for focal growth control. New Phytologist 229, 845860.

19. Kawai, Y., Ono, E., and Mizutani, M. (2014). Evolution and diversity of the 2oxoglutarate-dependent dioxygenase superfamily in plants. Plant $\mathrm{J} 78,328-343$.

20. Muff, S., Nilsen, E.B., O’Hara, R.B., and Nater, C.R. (2021). Rewriting results sections in the language of evidence. Trends in Ecology \& Evolution 0. 
21. Bennett, T.A., Liu, M.M., Aoyama, T., Bierfreund, N.M., Braun, M., Coudert, Y., Dennis, R.J., O'Connor, D., Wang, X.Y., White, C.D., et al. (2014). Plasma MembraneTargeted PIN Proteins Drive Shoot Development in a Moss. Curr. Biol. 24, 2776-2785.

22. Lang, D., Ullrich, K.K., Murat, F., Fuchs, J., Jenkins, J., Haas, F.B., Piednoel, M., Gundlach, H., Van Bel, M., Meyberg, R., et al. (2018). The Physcomitrella patens chromosome-scale assembly reveals moss genome structure and evolution. The Plant Journal 93, 515-533.

23. Romani, F. (2017). Origin of TAA Genes in Charophytes: New Insights into the Controversy over the Origin of Auxin Biosynthesis. Front. Plant Sci. 8, R899-3.

24. Delaux, P.-M., Hetherington, A.J., Coudert, Y., Delwiche, C., Dunand, C., Gould, S., Kenrick, P., Li, F.-W., Philippe, H., Rensing, S.A., et al. (2019). Reconstructing trait evolution in plant evo-devo studies. Curr. Biol. 29, R1110-R1118.

25. Eklund, D.M., Ishizaki, K., Flores-Sandoval, E., Kikuchi, S., Takebayashi, Y., Tsukamoto, S., Hirakawa, Y., Nonomura, M., Kato, H., Kouno, M., et al. (2015). Auxin Produced by the Indole-3-Pyruvic Acid Pathway Regulates Development and Gemmae Dormancy in the Liverwort Marchantia polymorpha. The Plant Cell Online 27, 1650-1669.

26. Solly, J.E., Cunniffe, N.J., and Harrison, C.J. (2017). Regional Growth Rate Differences Specified by Apical Notch Activities Regulate Liverwort Thallus Shape. Current Biology 27, 16-26.

27. Shubin, N., Tabin, C., and Carroll, S. (2009). Deep homology and the origins of evolutionary novelty. Nature $457,818-823$.

28. Véron, E., Vernoux, T., and Coudert, Y. (2020). Phyllotaxis from a Single Apical Cell. Trends in Plant Science.

29. Toyokura, K., Goh, T., Shinohara, H., Shinoda, A., Kondo, Y., Okamoto, Y., Uehara, T., Fujimoto, K., Okushima, Y., Ikeyama, Y., et al. (2019). Lateral Inhibition by a Peptide Hormone-Receptor Cascade during Arabidopsis Lateral Root Founder Cell Formation. Dev Cell 48, 64-75.e5.

30. Chandler, J.W., and Werr, W. (2017). DORNRÖSCHEN, DORNRÖSCHEN-LIKE, and PUCHI redundantly control floral meristem identity and organ initiation in Arabidopsis. $J$ Exp Bot 68, 3457-3472.

31. Capua, Y., and Eshed, Y. (2017). Coordination of auxin-triggered leaf initiation by tomato LEAFLESS. Proc Natl Acad Sci USA 114, 3246-3251.

32. Ishikawa, M., Morishita, M., Higuchi, Y., Ichikawa, S., Ishikawa, T., Nishiyama, T., Kabeya, Y., Hiwatashi, Y., Kurata, T., Kubo, M., et al. (2019). Physcomitrella STEMIN transcription factor induces stem cell formation with epigenetic reprogramming. Nat Plants 5, 681-690.

33. Fichtner, F., Barbier, F.F., Feil, R., Watanabe, M., Annunziata, M.G., Chabikwa, T.G., Höfgen, R., Stitt, M., Beveridge, C.A., and Lunn, J.E. (2017). Trehalose 6-phosphate is involved in triggering axillary bud outgrowth in garden pea (Pisum sativum L.). The Plant Journal 92, 611-623. 
34. Fichtner, F., Barbier, F.F., Annunziata, M.G., Feil, R., Olas, J.J., Mueller-Roeber, B., Stitt, M., Beveridge, C.A., and Lunn, J.E. (2021). Regulation of shoot branching in arabidopsis by trehalose 6-phosphate. New Phytol 229, 2135-2151.

35. Satoh-Nagasawa, N., Nagasawa, N., Malcomber, S., Sakai, H., and Jackson, D. (2006). A trehalose metabolic enzyme controls inflorescence architecture in maize. Nature 441, 227-230.

36. Claeys, H., Vi, S.L., Xu, X., Satoh-Nagasawa, N., Eveland, A.L., Goldshmidt, A., Feil, R., Beggs, G.A., Sakai, H., Brennan, R.G., et al. (2019). Control of meristem determinacy by trehalose 6-phosphate phosphatases is uncoupled from enzymatic activity. Nature Plants, 1-9.

37. Hiss, M., Meyberg, R., Westermann, J., Haas, F.B., Schneider, L., SchallenbergRüdinger, M., Ullrich, K.K., and Rensing, S.A. (2017). Sexual reproduction, sporophyte development and molecular variation in the model moss Physcomitrella patens: introducing the ecotype Reute. The Plant Journal 22, 9.

38. Thelander, M., Landberg, K., and Sundberg, E. (2019). Minimal auxin sensing levels in vegetative moss stem cells revealed by a ratiometric reporter. New Phytologist 224, 775-788.

39. Schaefer, D., Zryd, J.P., Knight, C.D., and Cove, D.J. (1991). Stable transformation of the moss Physcomitrella patens. Mol Gen Genet 226, 418-424.

40. Rensing, S.A., Lang, D., Zimmer, A.D., Terry, A., Salamov, A., Shapiro, H., Nishiyama, T., Perroud, P.-F., Lindquist, E.A., Kamisugi, Y., et al. (2008). The Physcomitrella genome reveals evolutionary insights into the conquest of land by plants. Science 319, 64-69.

41. Gagnot, S., Tamby, J.-P., Martin-Magniette, M.-L., Bitton, F., Taconnat, L., Balzergue, S., Aubourg, S., Renou, J.-P., Lecharny, A., and Brunaud, V. (2008). CATdb: a public access to Arabidopsis transcriptome data from the URGV-CATMA platform. Nucleic Acids Research 36, D986-D990.

42. Kopylova, E., Noé, L., and Touzet, H. (2012). SortMeRNA: fast and accurate filtering of ribosomal RNAs in metatranscriptomic data. Bioinformatics 28, 3211-3217.

43. Langmead, B., and Salzberg, S.L. (2012). Fast gapped-read alignment with Bowtie 2. Nat Methods 9, 357-359.

44. Rigaill, G., Balzergue, S., Brunaud, V., Blondet, E., Rau, A., Rogier, O., Caius, J., Maugis-Rabusseau, C., Soubigou-Taconnat, L., Aubourg, S., et al. (2018). Synthetic data sets for the identification of key ingredients for RNA-seq differential analysis. Briefings in

45. McCarthy, D.J., Chen, Y., and Smyth, G.K. (2012). Differential expression analysis of multifactor RNA-Seq experiments with respect to biological variation. Nucleic Acids Research 40, 4288-4297.

46. Heberle, H., Meirelles, G.V., da Silva, F.R., Telles, G.P., and Minghim, R. (2015). InteractiVenn: a web-based tool for the analysis of sets through Venn diagrams. BMC Bioinformatics 16, 169. 
47. Ge, S.X., Jung, D., and Yao, R. (2020). ShinyGO: a graphical gene-set enrichment tool for animals and plants. Bioinformatics 36, 2628-2629.

48. Edgar, R., Domrachev, M., and Lash, A.E. (2002). Gene Expression Omnibus: NCBI gene expression and hybridization array data repository. Nucleic Acids Research 30 , 207-210.

772 49. Kleiber, C., and Zeileis, A. (2020). AER: Applied Econometrics with R.

50. Ortiz-Ramírez, C., Hernandez-Coronado, M., Thamm, A., Catarino, B., Wang, M., Dolan, L., Feijó, J.A., and Becker, J.D. (2016). A Transcriptome Atlas of Physcomitrella patens Provides Insights into the Evolution and Development of Land Plants. Mol Plant 9, 205-220. 